\title{
Solvability and Stability of Impulsive Set Dynamic Equations on Time Scales
}

\author{
Shihuang Hong, Jing Gao, and Yingzi Peng \\ Science College, Hangzhou Dianzi University, Hangzhou 310018, China \\ Correspondence should be addressed to Shihuang Hong; hongshh@hotmail.com
}

Received 9 November 2013; Accepted 30 March 2014; Published 4 May 2014

Academic Editor: Shawn X. Wang

Copyright (c) 2014 Shihuang Hong et al. This is an open access article distributed under the Creative Commons Attribution License, which permits unrestricted use, distribution, and reproduction in any medium, provided the original work is properly cited.

\begin{abstract}
A class of new nonlinear impulsive set dynamic equations is considered based on a new generalized derivative of set-valued functions developed on time scales in this paper. Some novel criteria are established for the existence and stability of solutions of such model. The approaches generalize and incorporate as special cases many known results for set (or fuzzy) differential equations and difference equations when the time scale is the set of the real numbers or the integers, respectively. Finally, some examples show the applicability of our results.
\end{abstract}

\section{Introduction}

One of the most convenient generalizations of differential equations is the notion of set differential equations (SDEs). The main objects in this framework are set-valued functions of the form $U: J \rightarrow K_{c}^{n}$, where $J$ is an interval of real axis $\mathbb{R}$ and $K_{c}^{n}$ is the space of all nonempty compact and convex subsets of $\mathbb{R}^{n}$. The increasing need of theoretical models for the study of other problems emerging in optimal control theory, dynamic economy, and biological theory motivated the advance in SDEs. In the last decade, the study of SDEs has attracted the attention of many researches [1-28]. In [2123] the authors extended SDEs to set dynamic equations on time scales in order to unify such problems in the framework of set dynamic equations on a time scale.

Hukuhara derivative ( $H$-derivative, for short) of setvalued functions is the starting point for the topic of SDEs [29] and later also for fuzzy differential equations (FDEs) $[30]$. In $[17,18]$ the authors were concerned with the interrelation between SDEs and FDEs. However, Hukuhara differentiability concept has some drawbacks as pointed out in $[31,32]$. For instance, $H$-derivative depends on the existence of Hukuhara difference but the latter does not always exist. Recently, several generalized $H$-derivatives are proposed to overcome some shortcomings of this approach.
Let us mention that the strongly generalized differentiability (G-differentiability) was defined by considering lateral $\mathrm{H}$ derivatives (four cases) in [32]. In [33] the authors have introduced the concept of $g H$-differentiability, which is based on a generalization of the Hukuhara difference between two intervals. Chalco-Cano et al. in [34] defined $g H$ differentiability of set-valued functions from the real axis $\mathbb{R}$ into $K_{c}^{n}$ and studied its relationship to $G$-differentiability. In this paper we shall adopt $g$-differentiability of set-valued functions on time scales, which is corresponding in character with the above-mentioned $g H$-differentiability and is an extension of the $H$-derivative on time scales introduced by [21].

A number of processes in physics, biology, and control theory during their evolutionary development are subject to the action of short-time forces in the form of impulses. In most cases the duration of the action of these forces is negligibly small, as a result of which one can assume that the forces act only at certain moments of time. The impulsive differential equations represent a mathematical model of such processes. The theory of impulsive differential equations has attracted the attention of many scientists; see, for instance, $[18,20,22]$ and [35-38].

In the present paper, we investigative the existence and stability theory for impulsive set dynamic equations on 
time scales by introducing the notion of the exponential dichotomy and using fixed point theorems. Our main contribution of the paper lies in showing how the basic properties of exponential dichotomy theory for homogeneous linear set dynamic equations can be used to establish the existence and stability of nonlinear set dynamic equations on time scales, proposing the possibility to get some insight into and better understanding of the subtle difference between discrete and continuous systems and building a unified study framework of the corresponding problems.

The paper contains five sections. In Section 2 several basic definitions and properties of time scales and set-valued analysis are collected; especially, the $g$-derivative of setvalued functions on time scales is defined. Subsequently, in Section 3, the exponential dichotomy of the homogeneous linear set dynamic equation is introduced and the existence and uniqueness of solutions to a class of linear impulsive set dynamic equations under $g$-derivative of set-valued functions and the existence of bounded solutions to its nonlinear counterpart are presented. In Section 4 several preliminary results concerning stability are given by applying a fixed point theorem. In the final section, several examples are given to show the applicability of our main results.

\section{Preliminaries}

In this section, we recall briefly the necessary background material for a self-contained presentation of our study. We first recall the notion of the time scale built by Hilger and Bohner. For more details, we refer the reader to $[39,40]$.

A closed nonempty subset $\mathbb{T}$ of real axis $\mathbb{R}$ is called a time scale or measure chain. For $t \in \mathbb{T}$ we define the forward jump operator $\sigma: \mathbb{T} \rightarrow \mathbb{T}$ by $\sigma(t)=\inf \{\tau \in \mathbb{T}: \tau>t\}$, while the backward jump operator $\rho: \mathbb{T} \rightarrow \mathbb{T}$ is defined by $\rho(t)=$ $\sup \{\tau \in \mathbb{T}: \tau<t\}$. The function $\mu: \mathbb{T} \rightarrow[0, \infty)$ called the graininess function is defined by $\mu(t)=\sigma(t)-t$ for $t \in \mathbb{T}$. In this definition we put inf $\emptyset=\sup \mathbb{T}$ (i.e., $\sigma(t)=t$ if $\mathbb{T}$ has a maximum $t$ ) and $\sup \emptyset=\inf \mathbb{T}$ (i.e., $\rho(t)=t$ if $\mathbb{T}$ has a minimum $t$ ), where $\emptyset$ denotes the empty set. $t$ is said to be right scattered if $\sigma(t)>t$ and $t$ is said to be right dense (rd) if $\sigma(t)=t$. $t$ is said to be left scattered if $\rho(t)<t$ and $t$ is said to be left dense (ld) if $\rho(t)=t$. A point is said to be isolated (dense) if it is right scattered (right dense) and left scattered (left sense) at the same time. In this paper we stipulate that the time scale $\mathbb{T}$ is $\mathbb{\mathbb { V }}-\{M\}$ if $\mathbb{\mathbb { V }}$ has a left scattered maximum $M$.

We continue with a description of the basic known results for Hausdorff metrics, continuity, and differentiability for set-valued mappings on time scales and their corresponding properties within the framework of time scales. We refer readers to $[18,21]$ for details. The following operations can be naturally defined on it:

$$
\begin{gathered}
X+Y=\{x+y: x \in X, y \in Y\}, \\
\lambda X=\{\lambda x: x \in X\}, \quad \lambda \in \mathbb{R}, \\
X Y=\{x y: x \in X, y \in Y\} \quad \text { for } X, Y \in K_{c}^{n} .
\end{gathered}
$$

Here, assume that some product operation is defined on $\mathbb{R}^{n}$. The set $Z \in K_{c}^{n}$ satisfying $X=Y+Z$ is known as the geometric difference (Hukuhara difference) of the set $X$ and set $Y$ and is denoted by the symbol $X-{ }_{H} Y$. It is worthy to note that the geometric difference of two sets does not always exist but if it does, it is unique. A generalization of geometric difference proposed in [33] aims to guarantee the existence of difference for any two intervals in $K_{c}^{1}$. In the light of this, a generalized difference called the $g$-difference, "- $g$ ", can be defined for any $X, Y \in K_{c}^{n}$; that is,

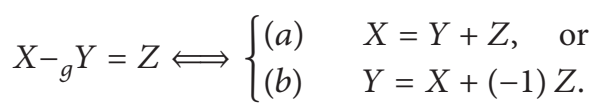

It is clear that if the $g$-difference exists, it is unique and it is a generalization of the geometric difference since $X-{ }_{g} Y=$ $X-{ }_{H} Y$, whenever $X-{ }_{H} Y$ exists. In addition, the authors in [33] enumerated the following properties.

Lemma 1. Let $X, Y \in K_{c}^{n}$ be two compact convex sets. Then,

(i) $X-{ }_{g} X=\{0\} ;(X+Y)-{ }_{g} Y=X ;\{0\}-{ }_{g}\left(X-{ }_{g} Y\right)=$ $(-Y)-{ }_{g}(-X)$

(ii) if $X{ }_{g} Y$ exists in the sense (a), then $Y-{ }_{g} X$ exists in the sense (b) and vice versa;

(iii) $X-{ }_{g} Y=Y-{ }_{g} X=Z$ if and only if $Z=\{0\}$ and $X=Y$;

(iv) $X-{ }_{g} Y$ exists if and only if $Y-{ }_{g} X$ and $(-Y)-{ }_{g}(-X)$ exist and $-\left(X-{ }_{g} Y\right)=(-Y)-{ }_{g}(-X)=Y-{ }_{g} X$.

Proof. (i)-(iii) for the proof we refer to [41]. To prove the first part of (iv) let $Z=X-{ }_{g} Y$; that is, $X=Y+Z$ or $Y=X+(-) Z$. Then $-X=-Y+(-Z)$ or $-Y=-X-(-Z)$ and this means $(-Y)-{ }_{g}(-X)=-Z$; the second part is immediate.

Throughout this paper, we always assume that the $g$ difference of any two elements under consideration in $K_{c}^{n}$ exists. We remark that the assumption may be valid; for instance, in the unidimensional case (with $K_{c}^{1}=\rrbracket$, a class of all closed bounded intervals of the real line) the $g$-difference exists for any two compact intervals.

We define the Hausdorff metric as

$$
D[X, Y]=\max \left\{\sup _{y \in Y} d(y, X), \sup _{x \in X} d(x, Y)\right\},
$$

where $d(x, Y)=\inf \{d(x, y): y \in Y\}$ and $X, Y$ are bounded subsets of $\mathbb{R}^{n}$.

Notice that $K_{c}^{n}$ with this distance is a complete metric space. On the other hand, the Hausdorff metric $D$ is compatible with the operations defined on it as described by the following properties: for any $X, Y, Z, W \in K_{c}^{n}$ and $\mu, \nu \in \mathbb{R}$,

$$
\begin{gathered}
D\left[X \pm_{g} Z, Y \pm_{g} Z\right]=D[X, Y], \\
D\left[X \pm_{g} Z, Y \pm_{g} W\right] \leq D[X, Y]+D[Z, W] ; \\
D[\mu X, \mu Y]=|\mu| D[X, Y], \\
D[X Z, Y Z] \leq\|Z\| D[X, Y] ; \\
D(\mu X, v X)=|\mu-\nu|\|X\| \quad \text { for } \mu, \nu \geq 0 \text { or } \mu, v \leq 0 .
\end{gathered}
$$

Here $\|V\|=D[V,\{0\}]=\sup \{\|v\|: v \in V\}$ for $V \in K_{c}^{n}$. 
In order to define the continuity and regularity of setvalued functions on time scales, we first need the notion of selectors of set-valued functions; that is, a function $f: \mathbf{D} \subset$ $\mathbb{T} \rightarrow \mathbb{R}$ is called a selector of the set-valued function $F$ : $\mathbf{D} \rightarrow K_{c}^{n}$ if $f(t) \in F(t)$ for all $t \in \mathbf{D}$.

Definition 2. A set-valued mapping $F: J_{\mathbb{T}} \rightarrow K_{c}^{n}$, where $J_{\mathbb{T}}=J \cap \mathbb{T}$ with $J \subset \mathbb{R}$, is said to have the limit at $t_{0} \in J_{\mathbb{T}}$ if there exists an element $\mathscr{A} \in K_{c}^{n}$ such that, for any $\varepsilon>0$, there exists a $\delta=\delta\left(\varepsilon, t_{0}\right)>0$ such that $D(F(t), \mathscr{A})<\varepsilon$, for all $t \in J_{\mathbb{T}}$ with $\left|t-t_{0}\right|<\delta$. We denote the limit by $\lim _{t \rightarrow t_{0}} F(x)$; that is, $\mathscr{A}=\lim _{t \rightarrow t_{0}} F(t)$.

Let $F\left(t_{0}\right)$ be well defined. $F$ is called continuous at $t_{0} \in J_{\mathbb{T}}$ if its limit at $t_{0}$ exists and equals $F\left(t_{0}\right)$.

$F: \mathbb{T} \rightarrow K_{c}^{n}$ is called regulated provided its right-sided limit exists at any right-dense point in $\mathbb{T}$, its left-sided limit exists at any left-dense point in $\mathbb{T}$, and its regulated selector exists.

$F$ is called right dense continuous, denoted $r d$ continuous, provided $F$ is continuous at each right-dense point in $\mathbb{T}$, its left-sided limits exist at each left-dense points in $\mathbb{T}$, and its $r d$-continuous selector exists. Similarly we can define $l d$-continuity.

$F$ is said to be uniformly $r d$-continuous on $\mathbf{D} \subset \mathbb{T}$ if it is $r d$-continuous and for any $\varepsilon>0$, there exists $\delta>0$ such that $D[F(t), F(s)]<\varepsilon$ for each right-dense point $t \in \mathbf{D}$ and any $s \in \mathrm{D}$ with $|s-t|<\delta$.

Lemma 3. Let the set-valued function $F:[a, b]_{\mathbb{T}} \rightarrow K_{c}^{n}$ be regulated, where $[a, b]_{\mathbb{T}}$ is a compact interval. Then $F$ is bounded; that is, there exists a positive number $M$ such that $\|F(t)\| \leq M$ for each $t \in[a, b]_{\mathbb{V}}$.

The following definition we refer to the $g H$ differentiability in [34] which can be regarded as an improvement of $H$-differentiability introduced in [21].

Definition 4. Suppose that $F: \mathbb{T} \rightarrow K_{c}^{n}$ is a set-valued function. Let $t \in \mathbb{T}$ and $\Delta_{g} F(t)$ be an element of $K_{c}^{n}$ (provided it exists) with the property that, for given any $\varepsilon>0$, there exists a neighborhood $U_{\mathbb{T}}$ of $t$ (i.e., $U_{\mathbb{T}}=(t-\delta, t+\delta) \cap \mathbb{T}$ for some $\delta>0$ ) such that

$$
D\left[F(t+h)-{ }_{g} F(\sigma(t)), \Delta_{g} F(t)(h-\mu(t))\right] \leq \varepsilon|h-\mu(t)|
$$

for all $t+h \in U_{\mathbb{T}}$ with $|h|<\delta$. We call $\Delta_{g} F(t)$ the $g$-derivative of $F$ at $t$.

We say that $F$ is $g$-differentiable at $t$ if its $g$-derivative exists at $t$. Moreover, we say $F$ is $g$-differentiable on $\mathbb{T}$ if its $g$-derivative exists at each $t \in \mathbb{T}$. The set-valued function $\Delta_{g} F: \mathbb{T} \rightarrow K_{c}^{n}$ is then called the $g$-derivative of $F$ on $\mathbb{T}$.

We denote the sets of all $r d$-continuous set-valued functions $F: \mathbf{D} \subset \mathbb{T} \rightarrow K_{c}^{n}$ and all set-valued functions $F: \mathbf{D} \rightarrow$ $K_{c}^{n}$ whose $r d$-continuous $g$-derivative exists, respectively, by

$$
\begin{aligned}
& \mathscr{C}_{r d}=\mathscr{C}_{r d}(\mathbf{D})=\mathscr{C}_{r d}\left(\mathbf{D}, K_{c}^{n}\right), \\
& \mathscr{C}_{r d}^{1}=\mathscr{C}_{r d}^{1}(\mathbf{D})=\mathscr{C}_{r d}^{1}\left(\mathbf{D}, K_{c}^{n}\right) .
\end{aligned}
$$

It is significant to refer that if we restrict ourselves to single valued mappings, then the previous notions reduce to their classical counterparts, that is, to ordinary $r d$-continuity and $\Delta$-differentiability in $\mathbb{T}$ (in the sense, $\Delta_{g} f(t)=f^{\Delta}(t)$ defined as [40] if $f$ is a single valued function). We enumerate the following properties for the $g$-differentiable set-valued functions.

Proposition 5. Assume that $F: \mathbb{T} \rightarrow K_{c}^{n}$ is a set-valued function and $t \in \mathbb{T}$; then we have the following.

(I) If $F$ is $g$-differentiable at $t$, then $F$ is continuous at $t$.

(II) If $F$ is continuous at $t$ and $t$ is right scattered, then $F$ is $g$-differentiable at $t$. Moreover, we have

$$
\Delta_{g} F(t)=\frac{F(\sigma(t))-{ }_{g} F(t)}{\mu(t)} .
$$

(III) If $t$ is right dense, then $F$ is $g$-differentiable at $t$ if and only if

$$
\lim _{h \rightarrow 0} \frac{F(t+h)-{ }_{g} F(t)}{h}=\Delta_{g} F(t) .
$$

Proof. (I) Assume that $F$ is $g$-differentiable at $t$. Let $\varepsilon \in(0,1)$. Define

$$
\varepsilon^{*}=\varepsilon\left[\left\|\Delta_{g} F(t)\right\|+2 \mu(t)+1\right]^{-1} .
$$

Clearly, $\varepsilon^{*} \in(0,1)$. Note that $D[X, Y] \leq\left\|X-{ }_{g} Y\right\|$ and by Definition 4 there exists a neighborhood $U_{\mathbb{T}}$ of $t$ such that

$$
D\left[F(t+h)-{ }_{g} F(\sigma(t)), \Delta_{g} F(t)(h-\mu(t))\right] \leq \varepsilon^{*}|h-\mu(t)|
$$

for all $h$ with $t+h \in U_{\mathbb{T}}$. Therefore, we have for all $t+h \epsilon$ $U_{\mathbb{T}} \cap\left(t-\varepsilon^{*}, t+\varepsilon^{*}\right)$ with $|h|<\varepsilon^{*}$

$$
\begin{aligned}
& D[F(t+h), F(t)] \\
& =D\left[F(t+h)-{ }_{g} F(\sigma(t))+F(\sigma(t))-{ }_{g} F(t),\right. \\
& \left.\quad \Delta_{g} F(t)(h-\mu(t))-{ }_{g} \Delta_{g} F(t)(h-\mu(t))\right] \\
& \leq D\left[F(t+h)-{ }_{g} F(\sigma(t)), \Delta_{g} F(t)(h-\mu(t))\right]
\end{aligned}
$$




$$
\begin{aligned}
& +D\left[F(\sigma(t))-{ }_{g} F(t),\{0\}-{ }_{g} \Delta_{g} F(t)(h-\mu(t))\right] \\
\leq & D\left[F(t+h)-{ }_{g} F(\sigma(t)), \Delta_{g} F(t)(h-\mu(t))\right] \\
& +D\left[F(\sigma(t))-{ }_{g} F(t), \Delta_{g} F(t) \mu(t)\right] \\
& +D\left[\Delta_{g} F(t) \mu(t),\{0\}-{ }_{g} \Delta_{g} F(t)(h-\mu(t))\right] \\
= & D\left[F(t+h)-{ }_{g} F(\sigma(t)), \Delta_{g} F(t)(h-\mu(t))\right] \\
& +D\left[F(\sigma(t))-{ }_{g} F(t), \Delta_{g} F(t) \mu(t)\right] \\
& +D\left[\Delta_{g} F(t) \mu(t), \Delta_{g} F(t)(\mu(t)-h)\right]
\end{aligned}
$$$$
\text { (see Lemma } 1 \text { (iv)) }
$$

$$
\begin{aligned}
\leq & \varepsilon^{*}|h-\mu(t)|+\varepsilon^{*} \mu(t)+|\mu(t)-(\mu(t)-h)|\left\|\Delta_{g} F(t)\right\| \\
\leq & \varepsilon^{*}(|h|+2 \mu(t)) \\
& +|h|\left\|\Delta_{g} F(t)\right\|<\varepsilon^{*}\left(1+2 \mu(t)+\left\|\Delta_{g} F(t)\right\|\right)=\varepsilon
\end{aligned}
$$

This implies that $F$ is continuous at $t$.

(II) Assume that $F$ is continuous at $t$ and $t$ is right scattered. By the continuity, we have

$$
\begin{aligned}
\lim _{h \rightarrow 0} \frac{F(t+h)-{ }_{g} F(\sigma(t))}{h-\mu(t)} & =\frac{F(t)-{ }_{g} F(\sigma(t))}{t-\sigma(t)} \\
& =\frac{F(t)-{ }_{g} F(\sigma(t))}{-\mu(t)} .
\end{aligned}
$$

This guarantees the existence of $\left(F(t)-{ }_{g} F(\sigma(t))\right) /-\mu(t)$. By virtue of Lemma 1(iv), the difference $\left(F(\sigma(t))-{ }_{g} F(t)\right) / \mu(t)$ exists and

$$
\frac{F(t)-{ }_{g} F(\sigma(t))}{-\mu(t)}=\frac{-\left(F(t)-{ }_{g} F(\sigma(t))\right)}{\mu(t)}=\frac{F(\sigma(t))-{ }_{g} F(t)}{\mu(t)} .
$$

Hence, given $\varepsilon>0$, there exists a neighborhood $U_{\mathbb{T}}$ of $t$ such that

$$
D\left[\frac{F(t+h)-{ }_{g} F(\sigma(t))}{h-\mu(t)}, \frac{F(\sigma(t))-{ }_{g} F(t)}{\mu(t)}\right] \leq \varepsilon
$$

for all $h$ with $t+h \in U_{\mathbb{T}}$. It follows that

$$
\begin{aligned}
& D\left[F(t+h)-{ }_{g} F(\sigma(t)), \frac{F(\sigma(t))-{ }_{g} F(t)}{\mu(t)}(h-\mu(t))\right] \\
& \quad \leq \varepsilon|h-\mu(t)|
\end{aligned}
$$

for all $h$ with $t+h \in U_{\mathbb{T}}$. According to Definition 4, (II) is valid as desired.

(III) Assume that $F$ is $g$-differentiable at $t$ and $t$ is right dense. Let $\varepsilon>0$ be given. Since $F$ is $g$-differentiable at $t$, there exists a neighborhood $U_{\mathbb{T}}$ of $t$ such that

$$
D\left[F(t+h)-{ }_{g} F(\sigma(t)), \Delta_{g} F(t)(h-\mu(t))\right] \leq \varepsilon|h-\mu(t)|
$$

for all $h$ with $t+h \in U_{\mathbb{T}}$. Since $\sigma(t)=t$, that is, $\mu(t)=0$, we have that

$$
D\left[F(t+h)-{ }_{g} F(t), h \Delta_{H} F(t)\right] \leq \varepsilon|h|
$$

for all $h$ with $t+h \in U_{\mathbb{T}}$. This yields

$$
D\left[\frac{F(t+h)-{ }_{g} F(t)}{h}, \Delta_{g} F(t)\right] \leq \varepsilon
$$

for all $h \neq 0$ with $t+h \in U_{\mathbb{T}}$. Therefore, from the arbitrariness of $\varepsilon$ we get

$$
\Delta_{g} F(t)=\lim _{h \rightarrow 0} \frac{F(t+h)-{ }_{g} F(t)}{h} .
$$

Conversely, suppose that $t$ is right dense, the limit exists in Part (III). Then, for any given $\varepsilon>0$, there is a neighborhood $U_{\mathbb{T}}$ of $t$ such that

$$
D\left[\frac{F(t+h)-{ }_{g} F(t)}{h}, \Delta_{g} F(t)\right] \leq \varepsilon
$$

for all $h \neq 0$ with $t+h \in U_{\mathbb{T}}$. This easily infers the desired result.

Proposition 6. Assume that set-valued functions $F, G: \mathbb{T} \rightarrow$ $K_{c}^{n}$ are $g$-differentiable; then one has the following.

(d1) The sum $F+G$ defined by $(F+G)(t)=F(t)+G(t)=$ $\{x+y: x \in F(t), y \in G(t)\}$ for each $t \in \mathbb{T}$ and the difference $F-{ }_{g} G$ defined by $\left(F-{ }_{g} G\right)(t)=F(t)-{ }_{g} G(t)$ are $g$-differentiable at $t \in \mathbb{T}$. Moreover,

$$
\begin{aligned}
& \Delta_{g}(F+G)(t)=\Delta_{g} F(t)+\Delta_{g} G(t), \\
& \Delta_{g}\left(F-{ }_{g} G\right)(t)=\Delta_{g} F(t)-{ }_{g} \Delta_{g} G(t) .
\end{aligned}
$$

(d2) For any constant $\lambda, \lambda F$ is $g$-differentiable at $t$ with

$$
\Delta_{g}(\lambda F)(t)=\lambda \Delta_{g} F(t) .
$$

(d3) The product function FG defined by $(F G)(t)=$ $F(t) G(t)$ for $t$ is $g$-differentiable at $t \in \mathbb{T}$ with

$$
\begin{aligned}
\Delta_{g}(F G)(t) & =F(\sigma(t)) \Delta_{g} G(t)+G(t) \Delta_{g} F(t) \\
& =F(t) \Delta_{g} G(t)+G(\sigma(t)) \Delta_{g} F(t) .
\end{aligned}
$$

Proof. (d1) Since $F$ and $G$ are $g$-differentiable at $t \in \mathbb{T}$, for any $\varepsilon>0$, there exist neighborhoods $U_{\mathbb{T}}$ and $V_{\mathbb{T}}$ of $t$ such that

$$
D\left[F(t+h)-{ }_{g} F(\sigma(t)), \Delta_{g} F(t)(h-\mu(t))\right] \leq \frac{\varepsilon}{2}|h-\mu(t)|
$$

for all $h$ with $t+h \in U_{\mathbb{T}}$ and

$$
D\left[G(t+h){ }_{g} G(\sigma(t)), \Delta_{g} G(t)(h-\mu(t))\right] \leq \frac{\varepsilon}{2}|h-\mu(t)|
$$


for all $h$ with $t+h \in V_{\mathbb{T}}$. Let $W_{\mathbb{T}}=U_{\mathbb{T}} \cap V_{\mathbb{T}}$. Then we have for all $h$ with $t+h \in W_{\mathbb{T}}$

$$
\begin{aligned}
& D\left[(F+G)(t+h)-{ }_{g}(F+G)(\sigma(t)),\right. \\
&\left.\left(\Delta_{g} F(t)+\Delta_{g} G(t)\right)(h-\mu(t))\right] \\
& \leq D\left[F(t+h)-{ }_{g} F(\sigma(t)), \Delta_{g} F(t)(h-\mu(t))\right] \\
& \quad+D\left[G(t+h)-{ }_{g} G(\sigma(t)), \Delta_{g} G(t)(h-\mu(t))\right] \\
& \leq \frac{\varepsilon}{2}|h-\mu(t)|+\frac{\varepsilon}{2}|h-\mu(t)|=\varepsilon|h-\mu(t)| .
\end{aligned}
$$

Therefore $F+G$ is $g$-differentiable at $t$ and $\Delta_{H}(F+G)(t)=$ $\Delta_{g} F(t)+\Delta_{g} G(t)$ as desired. The proof of the second formula is similar.

(d2) We assume that $\lambda \neq 0$. Otherwise, the desired result trivially holds. The differentiability of $F$ at $t \in \mathbb{T}$ guarantees that there exists the neighborhood $U_{\mathbb{T}}$ of $t$ such that, for any given $\varepsilon>0$, we have

$$
D\left[F(t+h)-{ }_{g} F(\sigma(t)), \Delta_{g} F(t)(h-\mu(t))\right] \leq \frac{\varepsilon}{|\lambda|}|h-\mu(t)|
$$

for all $h$ with $t+h \in U_{\mathbb{T}}$. This implies that

$$
\begin{aligned}
& D\left[\lambda F(t+h)-{ }_{g} \lambda F(\sigma(t)), \lambda \Delta_{g} F(t)(h-\mu(t))\right] \\
& \quad=|\lambda| D\left[F(t+h)-{ }_{g} F(\sigma(t)), \Delta_{g} F(t)(h-\mu(t))\right] \\
& \quad \leq|\lambda| \frac{\varepsilon}{|\lambda|}|h-\mu(t)|=\varepsilon|h-\mu(t)|
\end{aligned}
$$

for all $h$ with $t+h \in U_{\mathbb{T}}$. Therefore, $\lambda F$ is $g$-differentiable at $t$ and $\Delta_{g}(\lambda F)(t)=\lambda \Delta_{g} F(t)$ as desired.

(d3) Let $\varepsilon^{g} \in(0,1)$. Define $\varepsilon_{1}=$ $\varepsilon\left(1+\|G(t)\|+\|F(\sigma(t))\|+\left\|\Delta_{g} F(t)\right\|\right)^{-1}$. Then $\varepsilon_{1} \in(0,1)$ and there exist neighborhoods $U_{\mathbb{T}}^{1}, U_{\mathbb{T}}^{2}$, and $U_{\mathbb{T}}^{3}$ of $t$ such that

$$
D\left[F(t+h)-{ }_{g} F(\sigma(t)), \Delta_{g} F(t)(h-\mu(t))\right] \leq \varepsilon_{1}|h-\mu(t)|
$$

for all $h$ with $t+h \in U_{\mathbb{T}}^{1}$ and

$$
D\left[G(t+h)-{ }_{g} G(\sigma(t)), \Delta_{g} G(t)(h-\mu(t))\right] \leq \varepsilon_{1}|h-\mu(t)|
$$

for all $h$ with $t+h \in U_{\mathbb{T}}^{2}$. From Proposition 5(I) it follows that

$$
D[G(t+h), G(t)] \leq \varepsilon_{1}, \quad\|G(t+h)\| \leq\|G(t)\|+1
$$

for all $h$ with $t+h \in U_{\mathbb{T}}^{3}$. Let $U_{\mathbb{T}}=U_{\mathbb{T}}^{1} \cap U_{\mathbb{T}}^{2} \cap U_{\mathbb{T}}^{3}$ and $t+h \in U_{\mathbb{T}}$. Then

$$
\begin{aligned}
& D\left[(F G)(t+h){ }_{-g}(F G)(\sigma(t)),\right. \\
& \left.\left(F(\sigma(t)) \Delta_{g} G(t)+G(t) \Delta_{g} F(t)\right)(h-\mu(t))\right] \\
& \leq D\left[\left(G(t+h){ }_{g} G(\sigma(t))\right) F(\sigma(t)),\right. \\
& \left.F(\sigma(t)) \Delta_{g} G(t)(h-\mu(t))\right] \\
& +D\left[\left(F(t+h)-{ }_{g} F(\sigma(t))\right) G(t+h),\right. \\
& \left.G(t+h) \Delta_{g} F(t)(h-\mu(t))\right] \\
& +D\left[\theta, G(t) \Delta_{g} F(t)(h-\mu(t))\right. \\
& \left.{ }_{-g} G(t+h) \Delta_{g} F(t)(h-\mu(t))\right] \\
& \leq D\left[G(t+h){ }_{-g} G(\sigma(t)), \Delta_{g} G(t)(h-\mu(t))\right] \\
& \times\|F(\sigma(t))\| \\
& +D\left[F(t+h)-{ }_{g} F(\sigma(t)), \Delta_{g} F(t)(h-\mu(t))\right] \\
& \times\|G(t+h)\| \\
& +D[G(t+h), G(t)]\left\|\Delta_{g} F(t)\right\||h-\mu(t)| \\
& \leq \varepsilon_{1}\|F(\sigma(t))\||h-\mu(t)|+\varepsilon_{1}(\|G(t)\|+1)|h-\mu(t)| \\
& +\varepsilon_{1}\left\|\Delta_{g} F(t)\right\||h-\mu(t)| \\
& =\varepsilon_{1}\left(1+\|F(\sigma(t))\|+\|G(t)\|+\left\|\Delta_{g} F(t)\right\|\right)|h-\mu(t)| \\
& =\varepsilon|h-\mu(t)| \text {. }
\end{aligned}
$$

Thus, $\Delta_{g}(F G)(t)=F(\sigma(t)) \Delta_{g} G(t)+G(t) \Delta_{g} F(t)$. The second product rule follows from this last equation by interchanging the set-valued functions $F$ and $G$.

From [40] it follows that if a single valued function $g$ is $\Delta$-differentiable and $g^{\Delta}(t)=f(t)$, then we define the Cauchy integral by

$$
\int_{a}^{t} f(s) \Delta s=g(t)-g(a) .
$$

In this case, we say $f$ to be $\Delta$-integrable on interval $\mathbf{D}=$ $[a, t]_{\mathbb{T}} \subset \mathbb{T}$. In particular, by $\int_{a}^{\infty} f(t) \Delta t:=\lim _{b \rightarrow \infty} \int_{a}^{b} f(t) \Delta t$ we mean that $f$ is $\Delta$-integrable on $\mathbf{D}=[a, \infty)_{\mathbb{T}}$ provided this limit exists.

Similarly, we can introduce the integral of the set-valued functions. By $S_{F}(D)$ we mean the set of all $\Delta$-integrable selectors of $F$ on $\mathbf{D}$.

Definition 7 (see [21]). A set-valued function $F: \mathbb{T} \rightarrow K_{c}^{n}$ is called $\Delta_{g}$-integrable on $\mathbf{D} \subset \mathbb{T}$ if $F$ has at least a $\Delta$-integrable 
selector on D. In this case, we define the $\Delta_{g}$-integral of $F$ on D, denoted by $\int_{\mathbf{D}} F(s) \Delta s$, as the set

$$
\int_{\mathbf{D}} F(s) \Delta s=\left\{\int_{\mathbf{D}} f(s) \Delta s: f \in S_{F}(\mathbf{D})\right\} .
$$

Lemma 8 (see [21]). Assume that $t_{0}, T \in \mathbb{T}$, and $F, G$ : $\left[t_{0}, T\right]_{\mathbb{T}} \rightarrow K_{c}^{n}$ are $\Delta_{g}$-integrable and have $r d$-continuous selectors; then we have the following.

(i) $\int_{t_{0}}^{T}\left[F(s) \pm_{g} G(s)\right] \Delta s=\int_{t_{0}}^{T} F(s) \Delta s \pm_{g} \int_{t_{0}}^{T} G(s) \Delta s$.

(ii) $\int_{t_{0}}^{t} \lambda F(s) \Delta s=\lambda \int_{t_{0}}^{t} F(s) \Delta s, \lambda \in \mathbb{R}, t \in\left[t_{0}, T\right]_{\mathbb{T}}$.

(iii) $\int_{t_{0}}^{T} F(s) \Delta s=\int_{t_{0}}^{t} F(s) \Delta s+\int_{t}^{T} F(s) s \Delta s, t \in\left[t_{0}, T\right]_{\mathbb{T}}$ with $t_{0} \leq t \leq T$.

(iv) $\int_{t}^{\sigma(t)} F(s) \Delta s=\mu(t) F(t)$. Specially, $\int_{t}^{t} F(s) \Delta s=\{0\}$ for $t \in\left[t_{0}, T\right]_{\mathbb{T}}$.

(v) If $G \in \mathscr{C}_{r d}\left(\mathbb{T}, K_{c}^{n}\right)$, then, for any $t_{1}, t_{2} \in \mathbb{T}$, we have

$$
\int_{t_{1}}^{t_{2}} G(s) \Delta s=\{0\}-\int_{t_{2}}^{t_{1}} G(s) \Delta s .
$$

(vi) Let $G \in \mathscr{C}_{r d}^{1}\left(\mathbb{T} \times \mathbb{T}, K_{c}^{n}\right)$. If $\mathscr{F}(t)=\int_{t_{0}}^{t} G(t, s) \Delta s$, then

$$
\Delta_{g} \mathscr{F}(t)=G(\sigma(t), t)+\int_{t_{0}}^{t} \Delta_{g}^{t} G(t, s) \Delta s
$$

where $\Delta_{g}^{t} G(t, s)$ stands for the $g$-derivative of $G$ with respect to the first variable $t$.

(vii) If $f \in S_{F}\left(\left[t_{0}, T\right]_{\mathbb{T}}\right)$ implies that $f \in C_{r d}\left(\left[t_{0}, T\right]_{\mathbb{T}}\right)$, then $\|F(\cdot)\|:\left[t_{0}, T\right]_{\mathbb{T}} \rightarrow \mathbb{R}_{+}$is $\Delta$-integrable and

$$
\left\|\int_{t_{0}}^{T} F(s) \Delta s\right\| \leq \int_{t_{0}}^{T}\|F(s)\| \Delta s .
$$

(viii) If $f \in S_{F}\left(\left[t_{0}, T\right]_{\mathbb{T}}\right)$ and $g \in S_{G}\left(\left[t_{0}, T\right]_{\mathbb{T}}\right)$ imply that $f \in C_{r d}\left(\left[t_{0}, T\right]_{\mathbb{J}}\right)$ and $g \in C_{r d}\left(\left[t_{0}, T\right]_{\mathbb{J}}\right)$, respectively, then $D[F(\cdot), G(\cdot)]:\left[t_{0}, T\right]_{\mathbb{T}} \rightarrow \mathbb{R}_{+}$is $\Delta$-integrable and

$$
D\left[\int_{t_{0}}^{T} F(s) \Delta s, \int_{t_{0}}^{T} G(s) \Delta s\right] \leq \int_{t_{0}}^{T} D[F(s), G(s)] \Delta s .
$$

Proof. We only prove (vi). Let $\varepsilon>0$. By assumption there exists a neighborhood $U_{\mathbb{T}}^{1}$ of $t$ such that for all $h$ with $t+h \in U_{\mathbb{T}}^{1}$

$$
\begin{gathered}
D\left[G(t+h, s)-{ }_{g} G(\sigma(t), s), \Delta_{g}^{t} G(t, s)(h-\mu(t))\right] \\
\quad \leq \frac{\varepsilon}{3\left(t-t_{0}\right)}|h-\mu(t)| .
\end{gathered}
$$

Since $G$ is continuous at $(t, t)$, there exists a neighborhood of $t V_{\mathbb{T}}^{2}$ such that for $s \in U_{\mathbb{T}}^{2}$

$$
D(G(\sigma(t), s), G(\sigma(t), t))<\frac{\varepsilon}{3} .
$$

Now define $U_{\mathbb{T}}=U_{\mathbb{T}}^{1} \cap U_{\mathbb{T}}^{2}$ and let $h$ satisfy $|h|<$ $1,\left|\int_{t}^{t+h} \Delta_{g}^{t} G(t, s) \Delta s\right|<\varepsilon / 3$, and $t+h \in U_{\mathbb{T}}$. Then

$$
\begin{aligned}
& D\left[\mathscr{F}(t+h)-{ }_{g} \mathscr{F}(\sigma(t)),\left(G(\sigma(t), t)+\int_{t_{0}}^{t} \Delta_{g}^{t} G(t, s) \Delta s\right)\right. \\
& \times(h-\mu(t))] \\
& =D\left[\int_{t_{0}}^{t+h} G(t+h, s) \Delta s\right. \\
& -\int_{g} \int_{t_{0}}^{\sigma(t)} G(\sigma(t), s) \Delta s, G(\sigma(t), t)(h-\mu(t)) \\
& \left.+(h-\mu(t)) \int_{t_{0}}^{t} \Delta_{g}^{t} G(t, s) \Delta s\right] \\
& =D\left[\int_{t_{0}}^{t+h}\left(G(t+h, s)-{ }_{g} G(\sigma(t), s)\right) \Delta s\right. \\
& +\int_{\sigma(t)}^{t+h} G(\sigma(t), s) \Delta s, G(\sigma(t), t)(h-\mu(t)) \\
& +(h-\mu(t)) \int_{t_{0}}^{t+h} \Delta_{g}^{t} G(t, s) \Delta s \\
& \left.-_{g}(h-\mu(t)) \int_{t}^{t+h} \Delta_{g}^{t} G(t, s) \Delta s\right] \\
& \leq D\left[\int_{t_{0}}^{t+h}\left(G(t+h, s)-{ }_{g} G(\sigma(t), s)\right) \Delta s,\right. \\
& \left.(h-\mu(t)) \int_{t_{0}}^{t+h} \Delta_{g}^{t} G(t, s) \Delta s\right] \\
& +D\left[\int_{\sigma(t)}^{t+h} G(\sigma(t), s) \Delta s, G(\sigma(t), t)(h-\mu(t))\right. \\
& \left.-_{g}(h-\mu(t)) \int_{t}^{t+h} \Delta_{g}^{t} G(t, s) \Delta s\right] \\
& \leq D\left[\int_{t_{0}}^{t+h}\left(G(t+h, s)-{ }_{g} G(\sigma(t), s)\right) \Delta s,\right. \\
& \left.(h-\mu(t)) \int_{t_{0}}^{t+h} \Delta_{g}^{t} G(t, s) \Delta s\right] \\
& +D\left[\int_{\sigma(t)}^{t+h} G(\sigma(t), s) \Delta s, \int_{\sigma(t)}^{t+h} G(\sigma(t), t) \Delta s\right] \\
& +\left|(h-\mu(t)) \int_{t}^{t+h} \Delta_{g}^{t} G(t, s) \Delta s\right| \\
& \leq \mid \int_{t_{0}}^{t+h} D\left[G(t+h, s){ }_{g} G(\sigma(t), s),\right.
\end{aligned}
$$




$$
\begin{gathered}
\left.(h-\mu(t)) \Delta_{g}^{t} G(t, s)\right] \Delta s \mid \\
+\left|\int_{\sigma(t)}^{t+h} D[G(\sigma(t), s), G(\sigma(t), t)] \Delta s\right| \\
+|h-\mu(t)|\left|\int_{t}^{t+h} \Delta_{g}^{t} G(t, s) \Delta s\right| \\
<\frac{\varepsilon}{3}|h-\mu(t)|+\frac{\varepsilon}{3}|h-\mu(t)|+\frac{\varepsilon}{3}|h-\mu(t)|=\varepsilon .
\end{gathered}
$$

Here, we also have used Lemma 8 (viii).

Definition 9. A set-valued function $F: \mathbb{T} \rightarrow K_{c}^{n}$ is called predifferentiable with (region of differentiation) $\mathbf{D}$, provided $\mathrm{D} \subset \mathbb{T}, \mathbb{T} \backslash \mathbf{D}$ is countable and contains no right-scattered elements of $\mathbb{T}$, and $F$ is $g$-differentiable at each $t \in \mathbf{D}$.

Lemma 10. (i) Let $J \subset \mathbb{R}$ be an interval. If $t_{0} \in \mathbb{T}$, then $\mathscr{F}$ defined by

$$
\begin{array}{r}
\mathscr{F}(t)=X_{0}+\int_{t_{0}}^{t} F(s) \Delta s \quad \text { for } t \\
\in J_{\mathbb{T}}, X_{0} \in K_{c}^{n}, \\
F \in \mathscr{C}_{r d}\left(\mathbf{D}, K_{c}^{n}\right)
\end{array}
$$

is $g$-differentiable and one has

$$
\Delta_{g} \mathscr{F}(t)=F(t), \text { a.e. on } J_{\mathbb{T}} \text {. }
$$

(ii) If $F$ is $r d$-continuous and $t \in \mathbb{T}$, then

$$
\int_{t}^{\sigma(t)} F(s) \Delta s=\mu(t) F(t) .
$$

Proof. We only prove (i). Let $G(t)=\int_{t_{0}}^{t} F(s) \Delta s$. By virtue of Proposition 6(d1) $\Delta_{g} \mathscr{F}(t)$ exists and $\Delta_{g} \mathscr{F}(t)=\Delta_{g} G(t)$ provided $G(t)$ is $g$-differentiable. Thus, it is sufficient to check the $g$-differentiability of $G(t)$ and $\Delta_{g} G(t)=F(t)$.

It is evident that $F$ is regulated provided $F$ is $r d$ continuous. As similar argument to Theorems 8.12 and 8.13 in [40], we can show that the set-valued function $G(t)$ is predifferentiable with region of differentiation $\mathbf{D}$ such that $\Delta_{g} G(t)=F(t)$ for all $t \in \mathbf{D}$. If $t \in J_{\mathbb{T}} \backslash \mathbf{D}$, then $t$ is a rightdense point of $\mathbb{T}$. On the other hand, for nay $\varepsilon>0$, let $U_{\mathbb{T}}$ be a neighborhood of $t$ such that $\left|\int_{t}^{t+h} D[F(s), F(t)] \Delta s\right|<\varepsilon$ for all $h$ with $t+h \in U_{\mathbb{T}}$. So we have

$$
\begin{aligned}
D[G( & \left.t+h)-_{g} G(\sigma(t)), F(t)(h-\mu(t))\right] \\
& =D\left[\int_{t_{0}}^{t+h} F(s) \Delta s,-\int_{t_{0}}^{\sigma(t)} F(s) \Delta s, \int_{\sigma(t)}^{t+h} F(t) \Delta s\right] \\
& =D\left[\int_{\sigma(t)}^{t+h} F(s) \Delta s, \int_{\sigma(t)}^{t+h} F(t) \Delta s\right] \\
& \leq\left|\int_{\sigma(t)}^{t+h} D[F(s), F(t)] \Delta s\right| \\
& =\left|\int_{t}^{t+h} D[F(s), F(t)] \Delta s\right|<\varepsilon .
\end{aligned}
$$

This implies that $\Delta_{g} G(t)$ exists and $\Delta_{g} G(t)=F(t)$ on $J_{\mathbb{T}} \backslash \mathbf{D}$. This proof is complete.

Finally, we recall the concept of the matrix-valued functions introduced by [40]. An $m \times n$-matrix-valued function $A$ is said to be $\Delta$-differentiable on $\mathbb{T}$ provided each entry of $A$ is $\Delta$-differentiable on $\mathbb{T}$. In this case we put

$$
A^{\Delta}=\left(a_{i j}^{\Delta}\right)_{1 \leq i \leq m, i \leq j \leq n}, \quad \text { where } A=\left(a_{i j}\right) .
$$

An $n \times n$-matrix-valued function $A$ on $\mathbb{T}$ is called regressive provided

$$
I+\mu(t) A(t) \text { is invertible for all } t \in T .
$$

Here, $I$ stands for $n \times n$-identity matrix. The sets of regressive and $r d$-continuous matrix-valued functions will be denoted by $\mathscr{R}$. The set $\mathscr{R}_{1}^{+}$consists of all positively regressive and $r d$-continuous functions $p$ satisfying $1+\mu(t) p(t)>0$ for $t \in \mathbb{T}$. From now on, unless otherwise mentioned, the matrixvalued functions involved in equations are always assumed to belong to $\mathscr{R}$.

For $A, B \in \mathscr{R}$, the "circle plus" and "circle minus" of matrix-valued functions are referred to as, respectively,

$$
\begin{gathered}
(A \oplus B)(t)=A(t)+B(t)+\mu(t) A(t) B(t) \\
(A \ominus B)(t)=(A \oplus(\ominus B))(t) \quad \text { with } \\
(\ominus A)(t)=-[I+\mu(t) A(t)]^{-1} A(t)=-A(t)[I+\mu(t) A(t)]^{-1} .
\end{gathered}
$$

A matrix exponential function $e_{A}\left(t, t_{0}\right)$ is defined as a unique matrix-valued solution of the following initial value problem:

$$
Y^{\Delta}=A(t) Y, \quad Y\left(t_{0}\right)=I,
$$

where $A \in \mathscr{R}$ is an $n \times n$-matrix-valued function and $t_{0} \in \mathbb{T}$. Denote

$$
\begin{aligned}
\mathscr{A}=\left\{e_{B}(u, v): B \text { is an } n \times n\right. \text {-matrix-valued } \\
\text { function from } \mathbb{T} \text { into } \mathscr{R} \text { and } u, v \in \mathbb{T}\} .
\end{aligned}
$$


Assume $A$ is a constant $n \times n$ matrix. If $\mathbb{T}=\mathbb{R}$, then $e_{A}(u, v)=$ $e^{A(u-v)}$, while if $\mathbb{Z}=\mathbb{Z}$ and $I+A$ is invertible, then $e_{A}(u, v)=$ $(I+A)^{u-v}$, where $\mathbb{Z}$ stands for the integral number set and $e_{A}(u, v) \in \mathscr{A}$. In [40] the elements of $\mathscr{A}$ have been proved to possess the following properties.

Lemma 11. If $e_{A}(\cdot, \cdot), e_{B}(\cdot, \cdot) \in \mathscr{A}$, then

(i) $e_{0}(t, s) \equiv I, e_{A}(t, t) \equiv I, e_{A}(\sigma(t), s)=(I+$ $\mu(t) A(t)) e_{A}(t, s)$

(ii) $e_{A}(s, t)=e_{A}^{-1}(t, s)=e_{\ominus A^{*}}^{*}(t, s)$, where $A^{*}$ stands for the conjugate transpose of the matrix $A$;

(iii) $e_{A}(t, s) e_{A}(s, r)=e_{A}(t, r)$;

(iv) $e_{A \oplus B}(t, s)=e_{A}(t, s) e_{B}(t, s)$ if $e_{A}(t, s)$ and $B(t)$ commute;

(v) $e_{A}^{\Delta}(c, \cdot)=-e_{A}(c, \sigma(\cdot)) A$ for $c \in \mathbb{T}$;

(vi) $\int_{a}^{b} e_{A}(c, \sigma(t)) A(t) \Delta t=e_{A}(c, a)-e_{A}(c, b)$ for $a, b, c \in \mathbb{T}$.

\section{Solvability of ISDE}

We emphasize that $\mathbb{T}_{+}=\{t \in \mathbb{T} \mid t \geq 0\}$ and $F \in \mathscr{C}_{k}$ means that, at each point $(t, U) \in\left(t_{k-1}, t_{k}\right] \times \mathscr{C}_{r d}, F:\left(t_{k-1}, t_{k}\right] \times$ $\mathscr{C}_{r d} \rightarrow K_{c}^{n}$ is continuous if $t$ is a right-dense point and has the limit if $t$ is a left-dense point. Moreover, let $U_{t_{k}^{+}}=U\left(t_{k}^{+}\right)$ represent the right limit of $U(t)$ at $t_{k}$ if $t_{k}$ is right dense and $U_{t_{k}^{+}}=U\left(\sigma\left(t_{k}\right)\right)$ if $t_{k}$ is right scattered for $k=1,2, \ldots$ Let $P C\left[\mathbb{T}_{+} \times \mathscr{C}_{r d}, K_{c}^{n}\right]=\left\{F: \mathbb{T}_{+} \times \mathscr{C}_{r d} \rightarrow K_{c}^{n} \mid F \in \mathscr{C}_{k}\right.$ and $\lim _{(t, V) \rightarrow\left(t_{k}^{+}, U\right)} F(t, V)=F\left(t_{k}^{+}, U\right)$ exists for each $U \in \mathscr{C}_{r d}$ and $k=1,2, \ldots\}$, and $B C=B C\left[\mathbb{T}_{+}, K_{c}^{n}\right]=\left\{U \in P C\left[\mathbb{T}_{+}, K_{c}^{n}\right] \mid\right.$ $\|U(t)\|$ is bounded in $\left.\mathbb{T}_{+}\right\}$and $P C^{1}=P C^{1}\left[\mathbb{T}_{+}, K_{c}^{n}\right]=\{U \in$ $B C \mid U$ is $g$-differentiable in each interval $\left.\left(t_{k-1}, t_{k}\right)\right\}$. It is clear that $\left(B C, D_{0}\right)$ is a complete metric space if it is endowed with the distance $D_{0}(U, V)=\sup _{t \in \mathbb{T}_{+}} D(U(t), V(t))$.

Consider the impulsive set dynamic equation (ISDE)

$$
\begin{gathered}
\Delta_{g} U(t)=A(t) U(t)+F(t, U), \quad t \neq t_{k}, t \in \mathbb{T}_{+}, \\
U_{t_{k}^{+}}=J_{k} U\left(t_{k}\right), \quad t=t_{k} \in \mathbb{T}_{+}(k=0,1,2, \ldots), \\
U_{t_{0}}=U_{0} \in K_{c}^{n}, \quad t_{0} \in \mathbb{T}_{+},
\end{gathered}
$$

where $F \in P C\left[\mathbb{T}_{+} \times \mathscr{C}_{r d}, K_{c}^{n}\right], J_{k}: K_{c}^{n} \rightarrow K_{c}^{n}$ is a continuous linear operator; that is, for any $U, V \in K_{c}^{n}$, and $a, b \in \mathbb{R}$, one has $J_{k}\left(a U \pm_{g} b V\right)=a J_{k}(U) \pm_{g} b J_{k}(V)$, and $\left\{t_{k}\right\} \subset \mathbb{T}_{+}$is a sequence of points such that $0 \leq t_{0}<t_{1}<t_{2}<\cdots<t_{k}<\cdots$ and $\lim _{k \rightarrow \infty} t_{k}=\infty$. By a solution of ISDE (51) we mean that a set-valued function $U \in P C^{1}\left[\mathbb{T}_{+}, K_{c}^{n}\right]$ satisfies (51).

To explore the existence of solutions to ISDE, we introduce the exponential dichotomy of set dynamic equations. We define the product of a matrix and a subset in $\mathbb{R}^{n}$ as follows:

$$
A(t) U=\{A(t) u: u \in U\}
$$

for any $m \times n$-matrix-valued function $A(t)$ on $\mathbb{T}$ and subset $U \subset \mathbb{R}^{n}$.
By an analogue of the proofs of Theorems 5.24 and 5.27 in [40], respectively, together with the product rule of differential and Lemma 8 , it is easy to prove the following results.

Lemma 12. Let $A \in \mathscr{R}, F: \mathbb{T} \rightarrow K_{c}^{n}$ rd-conditions, $t_{0} \in \mathbb{T}$, and $U_{0} \in K_{c}^{n}$. Then the initial problem

$$
\begin{gathered}
\Delta_{g} U(t)=A(t) U(t)+F(t), \quad t \in \mathbb{T}, \\
U\left(t_{0}\right)=U_{0}
\end{gathered}
$$

with $F \in \mathscr{C}_{r d}\left(\mathbb{T}, K_{c}^{n}\right)$ having a unique solution $U: \mathbb{T} \rightarrow K_{c}^{n}$ given by

$$
\mathscr{U}_{0}\left(t, t_{0}, U_{0}\right)=e_{A}\left(t, t_{0}\right) U_{0}+\int_{t_{0}}^{t} e_{A}(t, \sigma(s)) F(s) \Delta s .
$$

Lemma 13. Let $A \in \mathscr{R}, F \in \mathscr{C}_{r d}\left(\mathbb{T}, K_{c}^{n}\right), t_{0} \in \mathbb{T}$, and $U_{0} \in K_{c}^{n}$. Then the initial problem

$$
\begin{gathered}
\Delta_{g} U(t)=-A^{*}(t) U(\sigma(t))+F(t), \quad t \in \mathbb{T} \\
U\left(t_{0}\right)=U_{0}
\end{gathered}
$$

has a unique solution $U: \mathbb{T}^{k} \rightarrow K_{c}^{n}$ given by

$$
\mathcal{U}_{0}^{*}\left(t, t_{0}, U_{0}\right)=e_{\ominus A^{*}}\left(t, t_{0}\right) U_{0}+\int_{t_{0}}^{t} e_{\ominus A^{*}}(t, s) F(s) \Delta s .
$$

In what follows, by means of $e_{A}(t, s) U_{s}$ we denote the unique solution $U\left(t, s, U_{s}\right)$ of the linear homogeneous set equation

$$
\begin{gathered}
\Delta_{g} U(t)=A(t) U(t), \quad t \in \mathbb{T}_{+}, \\
U(s)=U_{s} \in K_{c}^{n}
\end{gathered}
$$

with initial point $s \in \mathbb{T}_{+}$fixed. We call $e_{A}(t, s)$ the fundamental matrix of (57).

Definition 14. Equation (57) is said to admit an exponential dichotomy on $\mathbb{T}$ if there exist positive constants $k, a$ and a continuous projection (matrix) $P(s)$ (i.e., $P^{2}(s)=P(s)$ ) on $\mathbb{R}^{n}$ such that

$$
\begin{array}{r}
\left|e_{A}(t, s) P(s) e_{\ominus A^{*}}^{*}(\sigma(\tau), s)\right| \leq k e_{\ominus a}(t, \sigma(\tau)), \\
\tau, t \in \mathbb{T}_{+}, \quad t \geq \sigma(\tau) \geq s, \\
\left|e_{A}(t, s)(I-P(s)) e_{\ominus A^{*}}^{*}(\sigma(\tau), s)\right| \leq k e_{\ominus a}(\sigma(\tau), t), \\
\tau, t \in \mathbb{T}_{+}, \quad s \leq t \leq \tau,
\end{array}
$$

where $e_{A}(t, s)$ is the fundamental matrix of (57) and $|B|$ is the norm of the $n \times n$-matrix $B=\left(b_{i j}\right)_{n \times n}$, say, for example, $|B|=$ $\left(\sum_{i=1}^{n} \sum_{j=1}^{n}\left|b_{i j}\right|^{2}\right)^{1 / 2}$.

We need the following hypotheses.

$(\mathrm{H} 1) e_{A}(t, s)(I-P(s)) e_{\ominus A^{*}}^{*}(\tau, s) F(\tau)=e_{A}(t, s) e_{\ominus A^{*}}^{*}(\tau, s)$ $F(\tau)-{ }_{g} e_{A}(t, s) P(s) e_{\ominus A^{*}}^{*}(\tau, s) F(\tau)$ with any $t, \tau \in \mathbb{T}_{+}$. (In general, $(a-b) U \neq a U-{ }_{g} b U$ for $a, b \in \mathbb{R}$ and $\left.U \in K_{c}^{n}\right)$. 
(H2) There exists a constant $0<\chi<1$ such that $e_{a}\left(\sigma\left(t_{k}\right), \sigma\left(t_{k+1}\right)\right) \leq \chi$ for $k=0,1,2, \ldots$, where $a$ is given as in Definition 14.

Let $\mathscr{H}_{n}=\left\{\mathscr{H}=\left\{H_{m}\right\} \mid H_{m} \in K_{c}^{n}, \sup _{m \in \mathbb{N}}\left\|H_{m}\right\|<\infty\right\}$, $D_{\infty}\left(\mathscr{H}^{1}, \mathscr{H}^{2}\right)=\sup _{m \in \mathbb{N}} D\left(H_{m}^{1}, H_{m}^{2}\right)$ with $\mathscr{H}^{i}=\left\{H_{m}^{i}\right\} \in \mathscr{H}_{n}$ for $i=1,2$ and $\|\mathscr{H}\|_{\infty}=D_{\infty}(\mathscr{H}, \Theta)$, where $\Theta$ is the zero element of $\mathscr{H}_{n}$. It is easy to see that $\left(\mathscr{H}_{n}, D_{\infty}\right)$ is a complete metric space.

Theorem 15. Let $A \in \mathscr{R}$ be a $n \times n$-matrix-valued function, the linear homogenous set dynamic equation (57) with $s=t_{0}$ admits the exponential dichotomy on $\mathbb{T}_{+}$with positive constants $k, a$, and the projection $\mathrm{P}$ and the conditions (H1) and (H2) hold. If $F \in B C\left[\mathbb{T}_{+}, K_{c}^{n}\right]$ and $\mathscr{H}=\left\{H_{m}\right\} \in \mathscr{H}_{n}$, then the linear ISDE

$$
\begin{gathered}
\Delta_{g} U(t)=A(t) U(t)+F(t), \quad t \neq t_{k}(k=0,1,2, \ldots), \\
U_{t_{k}^{+}}=J_{k} U\left(t_{k}\right)+H_{k}, \quad k=0,1,2, \ldots, \\
U\left(t_{0}\right)=U_{0}
\end{gathered}
$$

has a unique bounded solution $\mathscr{U}(F, \mathscr{H})$ on $\mathbb{T}_{+}$satisfying

$$
\mathscr{U}(F, \mathscr{H})= \begin{cases}\mathscr{U}_{0}\left(t, t_{0}, U_{0}\right), & t \in\left[0, t_{0}\right]_{\mathbb{T}_{+}} \\ \mathscr{U}_{1}(t), & t \in\left(t_{0}, \infty\right)_{\mathbb{T}_{+}}\end{cases}
$$

with

$$
\begin{aligned}
\mathscr{U}_{1}(t)= & \int_{t_{0}}^{t} e_{A}^{0}(t) P e_{\ominus A^{*}}^{0}(\sigma(\tau)) F(\tau) \Delta \tau \\
& -\int_{g}^{\infty} e_{A}^{0}(t) P_{1} e_{\ominus A^{*}}^{0}(\sigma(\tau)) F(\tau) \Delta \tau \\
& +\sum_{t_{j}<t} e_{A}^{0}(t) P e_{\ominus A^{*}}^{0}\left(t_{j}^{+}\right) H_{j}-{ }_{g} \sum_{t_{j} \geq t} e_{A}^{0}(t) P_{1} e_{\ominus A^{*}}^{0}\left(t_{j}^{+}\right) H_{j}
\end{aligned}
$$

with $t \in \mathbb{T}_{+}, e_{A}^{0}(t)=e_{A}\left(t, t_{0}\right), e_{\ominus A^{*}}^{0}(t)=e_{\ominus A^{*}}^{*}\left(t, t_{0}\right), P=P\left(t_{0}\right)$ and $P_{1}=(I-P)$.

Proof. To show that the operator $\mathcal{U}: B C \times \mathscr{H}_{n} \rightarrow B C$ is continuous and $\mathscr{U}(F, \mathscr{H}) \in P C^{1}$, we shall first estimate the $\|\cdot\|$ of the addends in $(61)$ for $t \in\left(t_{0}, \infty\right)_{\mathbb{T}_{+}}$. Let $d$ be a positive constant such that $\|F(t)\| \leq d$ for all $t \in \mathbb{T}_{+}$. By Definition 14 and Lemma 8 (vii) we have

$$
\begin{aligned}
& \left\|\int_{t_{0}}^{t} e_{A}^{0}(t) P e_{\ominus A^{*}}^{0}(\sigma(\tau)) F(\tau) \Delta \tau\right\| \\
& \quad \leq \int_{t_{0}}^{t}\left\|e_{A}^{0}(t) P e_{\ominus A^{*}}^{0}(\sigma(\tau)) F(\tau)\right\| \Delta \tau
\end{aligned}
$$

$$
\begin{gathered}
\quad=\int_{t_{0}}^{t}\left|e_{A}^{0}(t) P e_{\ominus A^{*}}^{0}(\sigma(\tau))\right|\|F(\tau)\| \Delta \tau \\
\leq k \int_{t_{0}}^{t} e_{\ominus a}(t, \sigma(\tau))\|F(\tau)\| \Delta \tau \\
\quad=k e_{\ominus a}(t, 0) \int_{t_{0}}^{t} e_{\ominus a}(0, \sigma(\tau))\|F(\tau)\| \Delta \tau \\
\quad \leq k d \int_{0}^{t} e_{\ominus a}(0, \sigma(\tau)) \Delta \tau \leq \frac{k d}{a}, \\
\left\|\int_{t}^{\infty} e_{A}^{0}(t) P_{1} e_{\ominus A^{*}}^{0}(\sigma(\tau)) F(\tau) \Delta \tau\right\| \\
\quad \leq \int_{t}^{\infty}\left\|e_{A}^{0}(t) P_{1} e_{\ominus A^{*}}^{0}(\sigma(\tau)) F(\tau)\right\| \Delta \tau \\
\quad=\int_{t}^{\infty}\left|e_{A}^{0}(t) P_{1} e_{\ominus A^{*}}^{0}(\sigma(\tau))\right|\|F(\tau)\| \Delta \tau \\
\quad \leq k \int_{t}^{\infty} e_{\ominus a}(\sigma(\tau), t)\|F(\tau)\| \Delta \tau \\
\quad \leq k d e_{a}(t, 0) \int_{t}^{\infty} e_{a}(0, \sigma(\tau)) \Delta \tau \leq \frac{k d}{a}
\end{gathered}
$$

and analogously (noting that $e_{\ominus A^{*}}^{0}\left(t_{j}^{+}\right)=e_{\ominus A^{*}}^{0}\left(\sigma\left(t_{j}\right)\right)$ )

$$
\begin{aligned}
& \left\|\sum_{t_{j}<t} e_{A}^{0}(t) P e_{\ominus A^{*}}^{0}\left(t_{j}^{+}\right) H_{j}\right\| \\
& \quad \leq \sum_{t_{j}<t} k e_{\ominus a}\left(t, \sigma\left(t_{j}\right)\right)\left\|H_{j}\right\| \leq k\|\mathscr{H}\|_{\infty} \sum_{t_{j}<t} e_{\ominus a}\left(t, \sigma\left(t_{j}\right)\right), \\
& \left\|\sum_{t \leq t_{j}} e_{A}^{0}(t) P_{1} e_{\ominus A^{*}}^{0}\left(t_{j}^{+}\right) H_{j}\right\| \\
& \quad \leq \sum_{t \leq t_{j}} k e_{\ominus a}\left(\sigma\left(t_{j}\right), t\right)\left\|H_{j}\right\| \leq k\|\mathscr{H}\|_{\infty} \sum_{t \leq t_{j}} e_{\ominus a}\left(\sigma\left(t_{j}\right), t\right) .
\end{aligned}
$$

Assuming $t_{m} \leq t \leq t_{m+1}$ for some positive integer $m$, in virtue of $(\mathrm{H} 2)$, we have

$$
\begin{aligned}
& \sum_{t_{j}<t} e_{\ominus a}\left(t, \sigma\left(t_{j}\right)\right) \\
& \leq \sum_{j=0}^{m} e_{\ominus a}\left(\sigma\left(t_{m}\right), \sigma\left(t_{j}\right)\right) \\
&=\sum_{j=0}^{m} e_{a}\left(\sigma\left(t_{j}\right), \sigma\left(t_{j+1}\right)\right) e_{a}\left(\sigma\left(t_{j+1}\right), \sigma\left(t_{j+2}\right)\right) \\
& \quad \cdots e_{a}\left(\sigma\left(t_{m-1}\right), \sigma\left(t_{m}\right)\right)
\end{aligned}
$$




$$
\begin{aligned}
& \leq \sum_{j=0}^{m} \chi^{m-j} \leq \frac{1}{1-\chi} . \\
\sum_{t \leq t_{j}} e_{\ominus a}\left(\sigma\left(t_{j}\right), t\right) & \\
\leq & \sum_{j=0}^{\infty} e_{\ominus a}\left(\sigma\left(t_{j}\right), \sigma(0)\right) \\
= & \sum_{j=0}^{\infty} e_{a}\left(\sigma(0), \sigma\left(t_{1}\right)\right) e_{a}\left(\sigma\left(t_{1}\right), \sigma\left(t_{2}\right)\right) \\
& \quad \cdots e_{a}\left(\sigma\left(t_{j-1}\right), \sigma\left(t_{j}\right)\right) \\
\leq & e_{a}\left(\sigma(0), \sigma\left(t_{1}\right)\right) \sum_{j=0}^{\infty} \chi^{j-1}=\frac{1}{1-\chi} e_{a}\left(\sigma(0), \sigma\left(t_{1}\right)\right) .
\end{aligned}
$$

Substituting the above two inequalities into (63) and (64), respectively, we have

$$
\begin{gathered}
\left\|\sum_{t_{j}<t} e_{A}^{0}(t) P e_{\ominus A^{*}}^{0}\left(t_{j}^{+}\right) H_{j}\right\| \leq \frac{k}{1-\chi}\|\mathscr{H}\|_{\infty}, \\
\left\|\sum_{t \leq t_{j}} e_{A}^{0}(t) P_{1} e_{\ominus A^{*}}^{0}\left(t_{j}^{+}\right) H_{j}\right\| \leq \frac{k e_{a}\left(\sigma(0), \sigma\left(t_{1}\right)\right)}{1-\chi}\|\mathscr{H}\|_{\infty} .
\end{gathered}
$$

From (61) and (62) and (66) it follows that the set-valued function $\mathscr{U}(F, \mathscr{H})$ is bounded on $\left(t_{0}, \infty\right)_{\mathbb{W}_{+}}$. If $t \in\left[0, t_{0}\right]_{\mathbb{T}_{+}}$, from the exponential dichotomy it follows that $e_{A}^{0}(t)$ is bounded, say, $\left|e_{A}^{0}(t)\right| \leq c$ for $c>0$. Thus,

$$
\begin{aligned}
\|\mathscr{U}(F, \mathscr{H})(t)\| \\
=\left\|\mathcal{U}_{0}\left(t, t_{0}, U_{0}\right)\right\| \\
\quad=\left\|e_{A}^{0}(t)\left[U_{0}+\int_{t_{0}}^{t} e_{A}\left(t_{0}, \sigma(\tau)\right) F(\tau) \Delta \tau\right]\right\| \\
\quad \leq\left|e_{A}^{0}(t)\right|\left\|U_{0}\right\|+\left|e_{A}^{0}(t)\right| \int_{0}^{t_{0}}\left\|e_{A}\left(t_{0}, \sigma(\tau)\right) F(\tau)\right\| \Delta \tau \\
\quad \leq c\left[\left\|U_{0}\right\|+d c_{0}\right]
\end{aligned}
$$

with $c_{0}=\int_{0}^{t_{0}}\left|e_{A}\left(t_{0}, \sigma(\tau)\right)\right| \Delta \tau$. This implies that $\mathscr{U}(F, \mathscr{H})$ is bounded on $\left[0, t_{0}\right]_{\mathbb{T}_{+}}$, too. Consequently, $\mathscr{U}(F, \mathscr{H})$ is bounded on $\mathbb{T}_{+}$.

The continuity of $\mathscr{U}(F, \mathscr{H})$ for $t \neq t_{k}(k=0,1,2, \ldots)$ and the existence of the limit values $\mathscr{U}(F, \mathscr{H})\left(t_{k}^{+}\right)(k=0,1,2, \ldots)$ are immediately verified by Lemma 8 (vii); that is, $\mathscr{U}(F, \mathscr{H}) \in$ $B C$.

To verify that $\mathscr{U}(F, \mathscr{H}) \in P C^{1}$, by differentiating (61) for $t_{k} \neq t \in\left(t_{0}, \infty\right)_{\mathbb{T}_{+}}(k=0,1,2, \ldots)$ and taking into account Propositions 2.6(d1), (d2) and Lemmas 8(vii), (vi) and Lemma 11, we obtain

$$
\begin{aligned}
& \Delta_{g} \mathcal{U}(F, \mathscr{H})(t) \\
&=e_{A}^{0}(\sigma(t)) P e_{\ominus A^{*}}^{0}(\sigma(t)) F(t) \\
&+e_{A}^{0}(\sigma(t))(I-P) e_{\ominus A^{*}}^{0}(\sigma(t)) F(t) \\
&+\int_{t_{0}}^{t} A(t) e_{A}^{0}(t) P e_{\ominus A^{*}}^{0}(\sigma(\tau)) F(\tau) \Delta \tau \\
&-{ }_{g} \int_{t}^{\infty} A(t) e_{A}^{0}(t)(I-P) e_{\ominus A^{*}}^{0}(\sigma(\tau)) F(\tau) \Delta \tau \\
&+\sum_{t_{j}<t} A(t) e_{A}^{0}(t) P e_{\ominus A^{*}}^{0}\left(t_{j}^{+}\right) H_{j} \\
&-{ }_{g} \sum_{t_{j} \geq t} A(t) e_{A}^{0}(t) P_{1} e_{\ominus A^{*}}^{0}\left(t_{j}^{+}\right) H_{j} \\
&= F(t)+A(t) \mathcal{U}(F, \mathscr{H})(t), \quad t_{k} \neq t \in\left(t_{0}, \infty\right)_{\mathbb{T}_{+}} .
\end{aligned}
$$

In the case of $t \in\left[0, t_{0}\right)_{\mathbb{T}_{+}}$, observing $\mathscr{U}_{0}\left(t, t_{0}, U_{0}\right)=$ $e_{A}^{0}(t)\left[U_{0}+\int_{t_{0}}^{t} e_{A}\left(t_{0}, \sigma(\tau)\right) F(\tau) \Delta \tau\right]$ we have

$$
\begin{aligned}
& \Delta_{g} \mathcal{U}(F, \mathscr{H})(t) \\
&=\Delta_{g} \mathcal{U}_{0}\left(t, t_{0}, U_{0}\right) \\
&=A(t) e_{A}^{0}(t)\left[U_{0}+\int_{t_{0}}^{t} e_{A}\left(t_{0}, \sigma(\tau)\right) F(\tau) \Delta \tau\right] \\
&+e_{A}^{0}(\sigma(t)) e_{A}\left(t_{0}, \sigma(t)\right) F(t) \\
&= A(t) \mathcal{U}_{0}\left(t, t_{0}, U_{0}\right)+F(t) \\
&= A(t) \mathcal{U}(F, \mathscr{H})(t)+F(t) .
\end{aligned}
$$

This implies $\mathscr{U}(F, \mathscr{H}) \in P C^{1}$.

It remains to prove that $\mathscr{U}(F, \mathscr{H})$ satisfies (59). We have proved that the first equation of (59) is met for $t \neq t_{k}$. Consider system (51) with $F \equiv\{0\}$ and $U_{0}=V \in K_{c}^{n}$ if any given; it is not hard to see that this system has a unique solution $e_{A}^{0}(t) V$. According to the impulsive condition we obtain

$$
e_{A}^{0}\left(t_{k}^{+}\right) V=J_{k} e_{A}^{0}\left(t_{k}\right) V \quad \text { for } V \in K_{c}^{n}, k=0,1,2, \ldots
$$


Taking into account this and combining (H1), we obtain

$$
\begin{aligned}
& \mathcal{U}(F, \mathscr{H})\left(t_{k}^{+}\right) \\
& =\int_{t_{0}}^{t_{k}} e_{A}^{0}\left(t_{k}^{+}\right) P e_{\ominus A^{*}}^{0}(\sigma(\tau)) F(\tau) \Delta \tau \\
& { }_{-g} \int_{t_{k}}^{\infty} e_{A}^{0}\left(t_{k}^{+}\right) P_{1} e_{\ominus A^{*}}^{0}(\sigma(\tau)) F(\tau) \Delta \tau \\
& +\sum_{t_{j} \leq t_{k}} e_{A}^{0}\left(t_{k}^{+}\right) P e_{\ominus A^{*}}^{0}\left(t_{j}^{+}\right) H_{j} \\
& -g \sum_{t_{j}>t_{k}} e_{A}^{0}\left(t_{k}^{+}\right) P_{1} e_{\ominus A^{*}}^{0}\left(t_{j}^{+}\right) H_{j} \\
& =J_{k} \int_{t_{0}}^{t_{k}} e_{A}^{0}\left(t_{k}\right) P e_{\ominus A^{*}}^{0}(\sigma(\tau)) F(\tau) \Delta \tau \\
& -{ }_{g} J_{k} \int_{t_{k}}^{\infty} e_{A}^{0}\left(t_{k}\right) P_{1} e_{\ominus A^{*}}^{0}(\sigma(\tau)) F(\tau) \Delta \tau \\
& +J_{k} \sum_{t_{j}<t_{k}} e_{A}^{0}\left(t_{k}\right) P e_{\ominus A^{*}}^{0}\left(t_{j}^{+}\right) H_{j} \\
& -{ }_{g} J_{k} \sum_{t_{j} \geq t_{k}} e_{A}^{0}\left(t_{k}\right) P_{1} e_{\ominus A^{*}}^{0}\left(t_{j}^{+}\right) H_{j} \\
& +e_{A}^{0}\left(t_{k}^{+}\right) P e_{\ominus A^{*}}^{0}\left(t_{k}^{+}\right) H_{k}+e_{A}^{0}\left(t_{k}^{+}\right) P_{1} e_{\ominus A^{*}}^{0}\left(t_{k}^{+}\right) H_{k} \\
& =J_{k} \mathcal{U}(F, \mathscr{H})\left(t_{k}\right)+H_{k} \text {. }
\end{aligned}
$$

This shows that the second equation of (59) is met. The third equation of (59) is straightforwardly met. Conclusively, $\mathcal{U}(F, \mathscr{H})$ is a desired solution.

Finally, under the assumption of the exponential dichotomy, the zero solution of the linear homogeneous set dynamic equation $\Delta_{g} U(t)=A(t) U(t)$ is the unique solution that is bounded in $\mathbb{T}_{+}$(see, e.g., [42, Lemma 4.13]). Let $U_{1}, U_{2}$ both be the solutions of ISDE (59). From the first equation of (59) it follows that

$$
\begin{array}{r}
\Delta_{g} U_{1}(t)-{ }_{g} A(t) U_{1}(t)=\Delta_{g} U_{2}(t)-{ }_{g} A(t) U_{2}(t)= \\
F(t), \\
t \neq t_{k} .
\end{array}
$$

By Proposition 6(d1), this yields $\Delta_{g}\left(U_{1}(t)-{ }_{g} U_{2}(t)\right)=$ $A(t)\left(U_{1}(t)-{ }_{g} U_{2}(t)\right)$, which further shows that $U_{1}-{ }_{g} U_{2}$ is a solution of $\Delta_{g} U(t)=A(t) U(t)$. Hence, $U_{1}(t)-{ }_{g} U_{2}(t)=$ $\{0\}$; that is, $U_{1}(t)=U_{2}(t)$ for $t \neq t_{k}$. Since $U_{i}(i=1,2)$ is left continuous at $t_{k}$, we have $U_{1}\left(t_{k}\right)=\lim _{t \rightarrow t_{k}^{-}} U_{1}(t)=$ $\lim _{t \rightarrow t_{k}^{-}} U_{2}(t)=U_{2}\left(t_{k}\right)$ for $k=0,1,2, \ldots$. This guarantees the uniqueness of solutions and the proof is complete.
Corollary 16. Assume that the conditions of Theorem 15 are valid. If $F \in B C\left[\mathbb{T}_{+}, K_{c}^{n}\right]$, then ISDE

$$
\begin{gathered}
\Delta_{G} U(t)=A(t) U(t)+F(t), \quad t \neq t_{k}(k=0,1,2, \ldots), \\
U_{t_{k}^{+}}=J_{k} U\left(t_{k}\right), \quad k=0,1,2, \ldots, \\
U\left(t_{0}\right)=U_{0}
\end{gathered}
$$

has a unique bounded solution $\mathcal{U}(F, \mathscr{H})$ on $\mathbb{T}_{+}$satisfying

$\mathcal{U}(F, \mathscr{H})(t)$

$$
= \begin{cases}\mathscr{U}_{0}\left(t, t_{0}, U_{0}\right), & t \in\left[0, t_{0}\right]_{\mathbb{T}_{+}}, \\ \int_{t_{0}}^{t} e_{A}^{0}(t) P e_{\ominus A^{*}}^{0}(\sigma(\tau)) F(\tau) \Delta \tau & \\ -{ }_{g} \int_{t}^{\infty} e_{A}^{0}(t) P_{1} e_{\ominus A^{*}}^{0}(\sigma(\tau)) F(\tau) \Delta \tau, & t \in\left(t_{0}, \infty\right)_{\mathbb{T}_{+}}\end{cases}
$$

with $t \in \mathbb{T}_{+}^{k}$ and $P_{1}=(I-P)$. Especially, ISDE (73) except for the initial condition has a unique solution

$$
\begin{aligned}
\mathscr{U}(F, \mathscr{H})(t)= & \int_{0}^{t} e_{A}^{0}(t) P e_{\ominus A^{*}}^{0}(\sigma(\tau)) F(\tau) \Delta \tau \\
& -{ }_{g} \int_{t}^{\infty} e_{A}^{0}(t) P_{1} e_{\ominus A^{*}}^{0}(\sigma(\tau)) F(\tau) \Delta \tau .
\end{aligned}
$$

Proof. This is an immediate result by taking $H_{k}=0(k=$ $0,1,2, \ldots)$, that is, $\mathscr{H}=\Theta$, in Theorem 15 .

Remark 17. If we consider the complete metric space $B C_{0}=$ $B C_{0}\left[\mathbb{T}_{+}, K_{c}^{n}\right]$ consisting of the functions which belong to $B C$ and satisfy $U_{t_{k}^{+}}=J_{k}\left(U\left(t_{k}\right)\right)$, then, under the assumptions of Corollary 16 without the condition ( $\mathrm{H} 2)$, the result of Corollary 16 is still valid.

We are in a position to discuss the existence of solutions to nonlinear ISDE (51). We need the following well-known fixed point theorem which is the foundational tool to prove our main results.

Lemma 18. Let $f$ from $\left(K_{c}^{n}, D\right)$ into itself be a continuous and compact mapping. If the set

$$
\begin{gathered}
\mathscr{M}=\left\{A \in K_{c}^{n} \mid \text { there exists a constant } \lambda \in(0,1)\right. \\
\text { such that } A=\lambda f(A)\}
\end{gathered}
$$

is bounded; that is, there exists a positive constant $c$ such that $\|A\| \leq c$ for all $A \in \mathscr{M}$, then the operator $f$ has a fixed point in $\mathscr{C}=\left\{A \in K_{c}^{n}:\|A\| \leq c\right\}$.

Proof. Let $\mathscr{D}=\left\{A \in K_{c}^{n}:\|A\|<r\right\}$ with $r>0$. Then, by [18, Proposition 1.3.4], there exists a compact, convex set $\mathscr{N} \subset K_{c}^{n}$ such that $\mathscr{D}$ is an open subset of $\mathscr{N}$ and $\{0\} \in \mathscr{D}$. Suppose that $f$ has no fixed point on $\partial \mathscr{D}$ (otherwise we are finished) and $B \neq \lambda f(B)$ for all $B \in \partial \mathscr{D}$ and $\lambda \in[0,1]$. Consider

$$
\mathscr{E}=\{A \in \overline{\mathscr{D}}: A=t f(A) \text { for some } t \in[0,1]\} .
$$


Now $\mathscr{E} \neq \emptyset$ since $\{0\} \in \mathscr{D}$. In addition, the continuity of $f$ implies that $\mathscr{E}$ is closed. Note that $\mathscr{E} \cap \partial \mathscr{D}=\emptyset$, and therefore by Urysohn's lemma there exists a continuous function $\eta$ : $\overline{\mathscr{D}} \rightarrow[0,1]$ with $\eta(\mathscr{E})=1$ and $\eta(\partial \mathscr{D})=0$. Let

$$
g(A)= \begin{cases}\eta(A) f(A), & A \in \overline{\mathscr{D}}, \\ \{0\}, & A \in \mathcal{N} \backslash \overline{\mathscr{D}} .\end{cases}
$$

Now it is immediate that $g: \mathcal{N} \rightarrow \mathcal{N}$ is a continuous, compact map since $\eta, f$ are continuous and $f$ is compact. By Tychonoff's fixed point theorem, $g$ has a fixed point $A \in \mathcal{N}$. Note that $A \in \mathscr{E}$ and hence $A=g(A)=\eta(A) f(A)=f(A)$. As a result, $f$ has a fixed point in $\overline{\mathscr{D}}$.

Now consider $\mathscr{C}_{k}=\left\{A \in K_{c}^{n} \mid\|A\|<c+1 / k\right\}$. The above result guarantees that $f$ has a fixed point $A_{k} \in \overline{\mathscr{C}}_{k}$ for $k=1,2, \ldots$. In view of the compactness of $f$ there exists a subsequence $\left\{A_{k_{i}}\right\}$ of $\left\{A_{k}\right\}$ such that $f\left(A_{k_{i}}\right) \rightarrow A$; that is, $A_{k_{i}} \rightarrow A$. From the continuity of $f$ it follows that $A=f(A)$. Obviously, $A \in \mathscr{C}$.

Theorem 19. Suppose that (57) admits an exponential dichotomy on $\left(t_{0}, \infty\right)_{\mathbb{T}_{+}}$with positive constants $k, a$, a projection $P$, and the $n \times n$-matrix function $A$ which satisfy condition (H2), and $F \in P C\left[\mathbb{T}_{+} \times \mathscr{C}_{r d}, K_{k}^{n}\right]$ satisfies the following.

(i) For each $\Phi \in P C^{1}$, the set-valued function $F_{\Phi}(s)=$ $F(s, \Phi(s))$ satisfies the hypothesis (H1).

(ii) There exists a function $h:\left[0, t_{0}\right]_{\mathbb{T}_{+}} \rightarrow \mathbb{R}_{+}$satisfying $\sup _{t \in\left[0, t_{0}\right]_{\pi_{+}}} \int_{0}^{t}\left|e_{A}(t, \tau)\right| h(\tau) \Delta \tau<1$ such that

\section{$D(F(t, U), F(t, V))$}

$$
\leq h(t) D(U, V), \quad \forall t \in\left[0, t_{0}\right]_{\mathbb{T}_{+}}, U, V \in P C^{1} .
$$

(iii) There exists a function $\psi:\left(t_{0}, \infty\right)_{\mathbb{T}_{+}} \rightarrow \mathbb{R}_{+}$which is $\Delta$-integrable on $\left[t_{0}, \infty\right)_{\mathbb{T}}$ such that

$$
\begin{aligned}
& \|F(t, \Phi(t))\| \leq \psi(t), \\
& \quad \text { for each }(t, \Phi) \in\left(t_{0}, \infty\right)_{\mathbb{T}_{+}} \times B C, \quad t \neq t_{k} .
\end{aligned}
$$

Then nonlinear ISDE

$$
\begin{gathered}
\Delta_{G} U(t)=A(t) U(t)+F(t, U), \quad t \neq t_{k}(k=0,1,2, \ldots), \\
U_{t_{k}^{+}}=J_{k} U\left(t_{k}\right)+H_{k}, \quad k=0,1,2, \ldots, \\
U\left(t_{0}\right)=U_{0}
\end{gathered}
$$

has a bounded solution on $\mathbb{T}_{+}$denoted by

$$
U(t)= \begin{cases}e_{A}^{0}(t) U_{0} \\ \quad+\int_{t_{0}}^{t} e_{A}(t, \sigma(\tau)) F(\tau, U) \Delta \tau, \quad t \in\left[0, t_{0}\right]_{\mathbb{T}_{+}}, \\ \int_{t_{0}}^{t} e_{A}^{0}(t) P e_{\ominus A^{*}}^{0}(\sigma(\tau)) F(\tau, U) \Delta \tau \\ \quad-\int_{t}^{\infty} e_{A}^{0}(t) P_{1} e_{\ominus A^{*}}^{0} \\ \quad \times(\sigma(\tau)) F(\tau, U) \Delta \tau \\ \quad \sum_{t_{j}<t} e_{A}^{0}(t) P e_{\ominus A^{*}}^{0}\left(t_{j}^{+}\right) H_{j} \\ \quad-\sum_{g} \sum_{t_{j} \geq t}^{0} e_{A}^{0}(t) P_{1} e_{\ominus A^{*}}^{0}\left(t_{j}^{+}\right) H_{j}, & t \in\left(t_{0}, \infty\right)_{\mathbb{T}_{+}} .\end{cases}
$$

Proof. For any fixed $\Phi \in P C^{1}$, consider ISDE(81) with $F(t, \Phi)=F_{\Phi}(t)$ instead of $F(t, U)$, from Theorem 15 it follows that the linear ISDE has a unique solution $\mathcal{U}\left(F_{\Phi}, \mathscr{H}\right)$ such that $\mathcal{U}\left(F_{\Phi}, \mathscr{H}\right)=\delta_{1}(t) \mathcal{U}_{0}(t, \Phi)+\delta_{2}(t) \mathcal{U}_{1}(t, \Phi)$, where $\mathcal{U}_{0}(t, \Phi)=$ $e_{A}^{0}(t) U_{0}+\int_{t_{0}}^{t} e_{A}(t, \sigma(\tau)) F_{\Phi}(\tau) \Delta \tau, \mathscr{U}_{1}(t)$ is analogously given by (61) with $F$ replaced by $F_{\Phi}$, and

$$
\begin{aligned}
& \delta_{1}(t)= \begin{cases}1, & t \in\left[0, t_{0}\right]_{\mathbb{T}_{+}}, \\
0, & t \in\left(t_{0}, \infty\right)_{\mathbb{T}_{+}},\end{cases} \\
& \delta_{2}(t)= \begin{cases}0, & t \in\left[0, t_{0}\right]_{\mathbb{T}_{+}}, \\
1, & t \in\left(t_{0}, \infty\right)_{\mathbb{T}_{+}}\end{cases}
\end{aligned}
$$

Let $f(\Phi, \Psi)=f_{1}(\Phi)+f_{2}(\Psi)$ with $f_{1}(\Phi)(t)=\delta_{1}(t) \mathcal{U}_{0}(t, \Phi)$ and $f_{2}(\Psi)(t)=\delta_{2}(t) \mathcal{U}_{1}(t, \Psi)$. It is clear that $f$ is a map from $P C^{1} \times P C^{1}$ into $P C^{1}$ and any of its fixed point (i.e., there exists $\Gamma \in P C^{1}$ such that $\left.\Gamma=f(\Gamma, \Gamma)\right)$ is a solution of nonlinear ISDE (81). To this end, we have to prove that $f_{1}$ and $f_{2}$ have a fixed point in $P C^{1}$, respectively. We first observe that assumption (ii) guarantees $f_{1}$ to be a contractive mapping, and therefore, by Banach fixed point theorem, $f_{1}$ has a unique fixed point $\widehat{\Phi}_{1} \in P C^{1}\left[\left[0, t_{0}\right]_{\mathbb{T}_{+}}, K_{c}^{n}\right]$. Let $\widehat{\Phi}(t)$ be a set-valued function whose value is $\widehat{\Phi}_{1}(t)$ for $t \in\left[0, t_{0}\right]_{\mathbb{T}_{+}}$and $\{0\}$ for $t \in\left(t_{0}, \infty\right)_{\mathbb{T}_{+}}$. Then $\widehat{\Phi}$ is a unique fixed point of $f_{1}$ in $P C^{1}$.

We next prove $f_{2}$ has a fixed point in $P C^{1}$. As an analogue of the arguments of the proof of Theorem 15 , we easily see that $f_{2}$ is bounded and continuous on $P C^{1}$. We shall verify that $f_{2}$ is equicontinuous. In fact, for any $t_{1}, t_{2} \in\left(t_{0}, \infty\right)_{\mathbb{T}_{+}}$with $t_{1}<t_{2}$ and $\Phi \in P C^{1}$, in virtue of the properties of Hausdorff distance and Lemma 8 , combining our hypotheses, we have

$$
\begin{aligned}
& D\left(f_{2}(\Psi)\left(t_{2}\right), f_{2}(\Psi)\left(t_{1}\right)\right) \\
& \quad=D\left(\int_{t_{0}}^{t_{2}} e_{A}^{0}\left(t_{2}\right) P e_{\ominus A^{*}}^{0}(\sigma(\tau)) F_{\Psi}(\tau) \Delta \tau\right.
\end{aligned}
$$




$$
\begin{aligned}
& -{ }_{g} \int_{t_{2}}^{\infty} e_{A}\left(t_{2}\right) P_{1} e_{\ominus A^{*}}^{0}(\sigma(\tau)) F_{\Psi}(\tau) \Delta \tau \\
& +\sum_{t_{j}<t_{2}} e_{A}^{0}\left(t_{2}\right) P e_{\ominus A^{*}}^{0}\left(t_{j}^{+}\right) H_{j} \\
& -g \sum_{t_{j} \geq t_{2}} e_{A}^{0}\left(t_{2}\right) P_{1} e_{\ominus A^{*}}^{0}\left(t_{j}^{+}\right) H_{j}, \\
& \int_{t_{0}}^{t_{1}} e_{A}^{0}\left(t_{1}\right) P e_{\ominus A^{*}}^{0}(\sigma(\tau)) F_{\Psi}(\tau) \Delta \tau \\
& -\int_{t_{1}}^{\infty} e_{A}^{0}\left(t_{1}\right) P_{1} e_{\ominus A^{*}}^{0}(\sigma(\tau)) F_{\Psi}(\tau) \Delta \tau \\
& +\sum_{t_{j}<t_{1}} e_{A}^{0}\left(t_{1}\right) P e_{\ominus A^{*}}^{0}\left(t_{j}^{+}\right) H_{j} \\
& \left.-{ }_{g} \sum_{t_{j} \geq t_{1}} e_{A}^{0}\left(t_{1}\right) P_{1} e_{\ominus A^{*}}^{0}\left(t_{j}^{+}\right) H_{j}\right) \\
& =D\left(\int_{t_{0}}^{t_{1}} e_{A}^{0}\left(t_{2}\right) P e_{\ominus A^{*}}^{0}(\sigma(\tau)) F_{\Psi}(\tau) \Delta \tau\right. \\
& +\int_{t_{1}}^{t_{2}} e_{A}^{0}\left(t_{2}\right) P e_{\ominus A^{*}}^{0}(\sigma(\tau)) F_{\Psi}(\tau) \Delta \tau \\
& -\int_{t_{2}}^{\infty} e_{A}\left(t_{2}\right) P_{1} e_{\ominus A^{*}}^{0}(\sigma(\tau)) F_{\Phi}(\tau) \Delta \tau \\
& +\sum_{t_{j}<t_{2}} e_{A}^{0}\left(t_{2}\right) P e_{\ominus A^{*}}^{0}\left(t_{j}^{+}\right) H_{j} \\
& -g \sum_{t_{j} \geq t_{2}} e_{A}^{0}\left(t_{2}\right) P_{1} e_{\ominus A^{*}}^{0}\left(t_{j}^{+}\right) H_{j}, \\
& \int_{t_{0}}^{t_{1}} e_{A}^{0}\left(t_{1}\right) P e_{\ominus A^{*}}^{0}(\sigma(\tau)) F_{\Phi}(\tau) \Delta \tau \\
& -\int_{t_{1}}^{t_{2}} e_{A}^{0}\left(t_{1}\right) P_{1} e_{\ominus A^{*}}^{0}(\sigma(\tau)) F_{\Phi}(\tau) \Delta \tau \\
& -{ }_{g} \int_{t_{2}}^{\infty} e_{A}\left(t_{1}\right) P_{1} e_{\ominus A^{*}}^{0}(\sigma(\tau)) F_{\Phi}(\tau) \Delta \tau \\
& +\sum_{t_{j}<t_{1}} e_{A}^{0}\left(t_{1}\right) P e_{\ominus A^{*}}^{0}\left(t_{j}^{+}\right) H_{j} \\
& \left.-g \sum_{t_{j} \geq t_{1}} e_{A}^{0}\left(t_{1}\right) P_{1} e_{\ominus A^{*}}^{0}\left(t_{j}^{+}\right) H_{j}\right) \\
& \leq \mathscr{D}_{1}+\mathscr{D}_{2}+\mathscr{D}_{3}+\mathscr{D}_{4} \text {, }
\end{aligned}
$$

where

$$
\mathscr{D}_{1}=D\left(\int_{t_{0}}^{t_{1}} e_{A}^{0}\left(t_{2}\right) P e_{\ominus A^{*}}^{0}(\sigma(\tau)) F_{\Psi}(\tau) \Delta \tau,\right.
$$

$$
\begin{aligned}
& \left.\int_{t_{0}}^{t_{1}} e_{A}^{0}\left(t_{1}\right) P e_{\ominus A^{*}}^{0}(\sigma(\tau)) F_{\Psi}(s) \Delta s\right) \\
& \leq \int_{t_{0}}^{t_{1}} D\left[e_{A}^{0}\left(t_{2}\right) P e_{\ominus A^{*}}^{0}(\sigma(\tau)) F_{\Psi}(\tau),\right. \\
& \left.e_{A}^{0}\left(t_{1}\right) P e_{\ominus A^{*}}^{0}(\sigma(\tau)) F_{\Psi}(\tau)\right] \Delta \tau, \\
& \mathscr{D}_{2}=D\left(\int_{t_{1}}^{t_{2}} e_{A}^{0}\left(t_{2}\right) P e_{\ominus A^{*}}^{0}(\sigma(\tau)) F_{\Psi}(\tau) \Delta \tau,\right. \\
& \left.-{ }_{g} \int_{t_{1}}^{t_{2}} e_{A}^{0}\left(t_{1}\right) P_{1} e_{\ominus A^{*}}^{0}(\sigma(\tau)) F_{\Psi}(\tau) \Delta \tau\right) \\
& \leq \int_{t_{1}}^{t_{2}}\left[\left|e_{A}^{0}\left(t_{2}\right) P e_{\ominus A^{*}}^{0}(\sigma(\tau))\right|\right. \\
& \left.+\left|e_{A}^{0}\left(t_{1}\right) P_{1} e_{\ominus A^{*}}^{0}(\sigma(\tau))\right|\right]\left\|F_{\Psi}(\tau)\right\| \Delta \tau, \\
& \mathscr{D}_{3}=D\left(\int_{t_{2}}^{\infty} e_{A}^{0}\left(t_{2}\right) P_{1} e_{\ominus A^{*}}^{0}(\sigma(\tau)) F_{\Psi}(\tau) \Delta \tau,\right. \\
& \left.\int_{t_{2}}^{\infty} e_{A}^{0}\left(t_{1}\right) P_{1} e_{\ominus A^{*}}^{0}(\sigma(\tau)) F_{\Psi}(\tau) \Delta \tau\right) \\
& \leq \int_{t_{2}}^{\infty} D\left[e_{A}^{0}\left(t_{2}\right) P_{1} e_{\ominus A^{*}}^{0}(\sigma(\tau)) F_{\Psi}(\tau),\right. \\
& \left.e_{A}^{0}\left(t_{1}\right) P_{1} e_{\ominus A^{*}}^{0}(\sigma(\tau)) F_{\Psi}(\tau)\right] \Delta \tau, \\
& \mathscr{D}_{4}=D\left(\sum_{t_{j}<t_{2}} e_{A}^{0}\left(t_{2}\right) P e_{\ominus A^{*}}^{0}\left(t_{j}^{+}\right) H_{j}\right. \\
& -g \sum_{t_{j} \geq t_{2}} e_{A}^{0}\left(t_{2}\right) P_{1} e_{\ominus A^{*}}^{0}\left(t_{j}^{+}\right) H_{j}, \\
& \sum_{t_{j}<t_{1}} e_{A}^{0}\left(t_{1}\right) P e_{\ominus A^{*}}^{0}\left(t_{j}^{+}\right) H_{j} \\
& \left.-{ }_{g} \sum_{t_{j} \geq t_{1}} e_{A}^{0}\left(t_{1}\right) P_{1} e_{\ominus A^{*}}^{0}\left(t_{j}^{+}\right) H_{j}\right) .
\end{aligned}
$$

Let $t_{2} \rightarrow t_{1}$, and from assumption (iii), together with the continuity of $e_{A}^{0}(t)$ and $\Delta$-integrable of $\psi$, it follows that $f_{2}$ is equicontinuous with respect to $\Psi \in P C^{1}$. In virtue of AscoliArzela theorem we obtain that $f_{2}$ is a continuous compact operator.

Now we prove that the set $\mathscr{M}$ defined by (76) is bounded. If it is contrary, there exist $\left(\lambda_{m}, U_{m}\right) \in(0,1) \times P C^{1}$ such that $U_{m}=\lambda_{m} f_{2}\left(U_{m}\right)$ and $\left\|U_{m}\right\|_{0}>m$ for $m=1,2 \ldots$. On the other hand, for any $t \in\left(t_{0}, \infty\right)_{\mathbb{W}_{+}}$, from (61) and (66), Definition 14, and assumption (iii) we have 


$$
\begin{aligned}
& \left\|U_{m}(t)\right\| \\
& \leq \lambda_{m} \int_{t_{0}}^{t}\left\|e_{A}^{0}(t) P e_{\ominus A^{*}}^{0}(\sigma(\tau)) F_{U_{m}}(\tau)\right\| \Delta \tau \\
& +\lambda_{m} \int_{t}^{\infty}\left\|e_{A}^{0}(t) P_{1} e_{\ominus A^{*}}^{0}(\sigma(\tau)) F_{U_{m}}(\tau)\right\| \Delta \tau \\
& +\lambda_{m}\left\|\sum_{t_{j}<t} e_{A}^{0}(t) P e_{\ominus A^{*}}^{0}\left(t_{j}^{+}\right) H_{j}\right\| \\
& +\lambda_{m}\left\|\sum_{t_{j} \geq t} e_{A}^{0}(t, 0) P_{1} e_{\ominus A^{*}}^{0}\left(t_{j}^{+}\right) H_{j}\right\| \\
& \leq k \int_{0}^{t} e_{\ominus a}(t, \sigma(\tau))\left\|F_{U_{m}}(\tau)\right\| \Delta \tau \\
& +k \int_{t}^{\infty} e_{\ominus a}(\sigma(\tau), t)\left\|F_{U_{m}}(\tau)\right\| \Delta \tau \\
& +\frac{k}{1-\chi}\|\mathscr{H}\|_{\infty}+\frac{k e_{a}\left(\sigma(0), \sigma\left(t_{1}\right)\right)}{1-\chi}\|\mathscr{H}\|_{\infty} \\
& \leq k \int_{0}^{t} e_{\ominus a}(t, \sigma(s)) \psi(s) \Delta s \\
& +k \int_{t}^{\infty} e_{\ominus a}(\sigma(s), t) \psi(s) \Delta s \\
& +\frac{k}{1-\chi}\|\mathscr{H}\|_{\infty}+\frac{k e_{a}\left(\sigma(0), \sigma\left(t_{1}\right)\right)}{1-\chi}\|\mathscr{H}\|_{\infty} \\
& \leq 2 k \int_{0}^{\infty} \psi(\tau) \Delta \tau+\frac{k}{1-\chi}\|\mathscr{H}\|_{\infty} \\
& +\frac{k e_{a}\left(\sigma(0), \sigma\left(t_{1}\right)\right)}{1-\chi}\|\mathscr{H}\|_{\infty}
\end{aligned}
$$

in view of the arbitrariness of $t \in\left(t_{0}, \infty\right)_{\mathbb{T}_{+}}$, we obtain

$$
\begin{aligned}
\left\|U_{m}\right\|_{0} \leq & 2 k \int_{0}^{\infty} \psi(\tau) \Delta \tau+\frac{k}{1-\chi}\|\mathscr{H}\|_{\infty} \\
& +\frac{k e_{a}\left(\sigma(0), \sigma\left(t_{1}\right)\right)}{1-\chi}\|\mathscr{H}\|_{\infty} .
\end{aligned}
$$

Let us take $m$ to be large enough, say, larger than the right hand side of the above inequality; we have a contradiction. Consequently, the set $\mathscr{M}$ is bounded. Lemma 18 guarantees that $f_{2}$ has a fixed point $\widehat{\Psi}_{1} \in P C^{1}\left[\left(t_{0}, \infty\right)_{\mathbb{T}_{+}}, K_{c}^{n}\right]$. Set a set-valued function $\widehat{\Psi}(t)$ such that its value is $\widehat{\Psi}_{1}(t)$ for $t \epsilon$ $\left(t_{0}, \infty\right)_{\mathbb{T}_{+}}$and $\{0\}$ for $t \in\left[0, t_{0}\right]_{\mathbb{T}_{+}}$. Then $\widehat{\Psi}$ is a fixed point of $f_{2}$ in $P C^{1}$.

We finally prove that $f$ has a fixed point in $P C^{1} \times P C^{1}$. Let $\Psi \in P C^{1}$ be fixed and define the mapping

$$
G(\Psi)=f(\Phi, \Psi)=f_{1}(\Phi)+f_{2}(\Psi) .
$$

Then from the fact that $f_{1}$ has a unique fixed point $\widehat{\Phi}$ satisfying $\widehat{\Phi}(t)=\{0\}$ for $t \in\left(t_{0}, \infty\right)_{\mathbb{T}_{+}}$and $f_{2}(\Psi)(t)=\{0\}$ for $t \in\left[0, t_{0}\right]_{\mathbb{T}_{+}}$, it follows that $\widehat{\Phi}+f_{2}(\Psi)=G(\Psi)$. Similarly, we can check that $G: P C^{1} \rightarrow P C^{1}$ meets all conditions of Lemma 18; therefore, $G$ has a fixed point $\Upsilon \in P C^{1}$ satisfying $\Upsilon(t)=\widehat{\Phi}(t)$ for $t \in\left[0, t_{0}\right]_{\mathbb{T}_{+}}$. This further implies that $\Upsilon=$ $f(\widehat{\Phi}, \Upsilon)=f(\Upsilon, \Upsilon)$; that is, $f$ has a fixed point in $P C^{1}$. This completes the proof.

Corollary 20. Assume that all conditions of Theorem 19 are satisfied, except for (H2). In addition, instead of $P C^{1}$ we consider the complete metric space $P C^{0}\left[\mathbb{T}_{+}, K_{c}^{n}\right]$ consisting of the functions $U \in B C_{0}$ and their g-derivatives exist; then nonlinear ISDE (51) has at least bounded solution.

\section{Some Stability Criteria}

In this section, we assume that $F(t,\{0\}) \equiv\{0\}, J_{k}(\{0\})+H_{k}=$ $\{0\}$ for $t \in \mathbb{T}_{+}$and $k=0,1,2, \ldots$ Moreover, by $F \in \operatorname{Lip}(h)$ we mean that $F \in P C\left[\mathbb{T}_{+} \times \mathscr{C}_{r d}, K_{k}^{n}\right]$ and

$$
\begin{aligned}
& D(F(t, U), F(t, V)) \\
& \quad \leq h(t) D(U, V), \quad \forall t \in \mathbb{T}_{+}, U, V \in P C^{1},
\end{aligned}
$$

where the function $h: \mathbb{T}_{+} \rightarrow \mathbb{R}_{+}$satisfies $k(1+a \mu(t)) h(t)<a$ and $\int_{0}^{\infty} k(1+a \mu(\tau)) h(\tau) \Delta \tau<\infty$ with the constants $k$, $a$ given as in Definition 14 and $\mu$ a graininess function. For the sake of convenience, we assume the projection $P \equiv I$.

Under the assumptions of $F \in \operatorname{Lip}(h)$, employing the procedure used in the proof of Theorem 19, we can obtain that the set dynamic equation

$$
\begin{gathered}
\Delta_{g} U(t)=A(t) U(t)+F(t, U), \quad t \in I_{k}=\left(t_{k}, t_{k+1}\right]_{T_{+}}, \\
U_{t_{k}^{+}}=\Phi_{k}=J_{k}\left(U\left(t_{k}\right)\right)+H_{k}
\end{gathered}
$$

has a solution

$$
\begin{aligned}
V_{k}(t) & =V_{k}\left(t_{k}, \Phi_{k}\right)(t) \\
& =e_{A}\left(t, t_{k}^{+}\right) \Phi_{k}+\int_{t_{k}}^{t} e_{A}(t, \sigma(\tau)) F\left(\tau, V_{k}\right) \Delta \tau, \quad t \in I_{k}
\end{aligned}
$$

for $k=0,1,2, \ldots$ Additionally define $V_{0}\left(t_{0}\right)=U_{0}, V_{k}\left(t_{k}\right)=$ $V_{k-1}\left(t_{k}\right)$ for $k=1,2, \ldots$. We now obtain a solution of ISDE (81) $U\left(t_{0}, U_{0}\right)$ on $\mathbb{T}_{+}$which is left continuous on $I_{k}$ and defined by

$$
U\left(t_{0}, U_{0}\right)(t)= \begin{cases}\mathcal{U}_{0}\left(t, t_{0}, U_{0}\right), & t \in\left[0, t_{0}\right]_{\mathbb{T}_{+}}, \\ V_{0}(t), & t \in I_{0}, \\ \vdots & \vdots \\ V_{k}(t), & t \in I_{k} . \\ \vdots & \vdots\end{cases}
$$


On the other hand, with the additional assumption that $P \equiv I$, from (82) we obtain a bounded solution of ISDE (81)

$$
\bar{U}(t)=\left\{\begin{array}{cc}
e_{A}^{0}(t) U_{0} & \\
+\int_{t_{0}}^{t} e_{A}(t, \sigma(\tau)) F(\tau, \bar{U}) \Delta \tau, & t \in\left[0, t_{0}\right]_{\mathbb{T}_{+}}, \\
\int_{t_{0}}^{t} e_{A}^{0}(t) e_{\ominus A^{*}}^{0}(\sigma(\tau)) F(\tau, \bar{U}) \Delta \tau & \\
+\sum_{t_{j}<t} e_{A}^{0}(t) e_{\ominus A^{*}}^{0}\left(t_{j}^{+}\right) H_{j}, & t \in\left(t_{0}, \infty\right)_{\mathbb{T}_{+}} .
\end{array}\right.
$$

Since $e_{A}^{0}(t) e_{\ominus A^{*}}^{0}(\sigma(\tau))=e_{A}\left(t, t_{0}\right) e_{A}\left(t_{0}, \sigma(\tau)\right)=e_{A}(t, \sigma(\tau))$, subtracting $\bar{U}$ from $V_{k}$, we have

$$
\begin{aligned}
V_{k}(t)-{ }_{g} \bar{U}(t) \\
=e_{A}\left(t, t_{k}^{+}\right) \Phi_{k}+\int_{t_{k}}^{t} e_{A}(t, \sigma(\tau)) F\left(\tau, V_{k}\right) \Delta \tau \\
\quad-\int_{t_{0}}^{t} e_{A}^{0}(t) e_{\ominus A^{*}}^{0}(\sigma(\tau)) F(\tau, \bar{U}) \Delta \tau \\
+\sum_{t_{j}<t} e_{A}^{0}(t) e_{\ominus A^{*}}^{0}\left(t_{j}^{+}\right) H_{j} \\
=e_{A}\left(t, t_{k}^{+}\right) \Phi_{k}+\int_{t_{k}}^{t} e_{A}(t, \sigma(\tau)) \\
\quad+\sum_{t_{j}<t} e_{A}^{0}(t) e_{\ominus A^{*}}^{0}\left(t_{j}^{+}\right) H_{j} .
\end{aligned}
$$

Thus, we have the following attractive result.

Theorem 21. Suppose that (57) admits an exponential dichotomy on $\left(t_{0}, \infty\right)_{\mathbb{T}_{+}}$with positive constants $k, a$, the projection $P=I$, and the $n \times n$-matrix function $A$ which satisfy the condition (H2), and $F \in \operatorname{Lip}(h)$. In addition, if

(i) for each $\Phi \in P C^{1}$, the set-valued function $F_{\Phi}(s)=$ $F(s, \Phi(s))$ satisfies the hypothesis (H1),

(ii) there exists $l>0$ such that $\left\|\Phi_{k}\right\| \leq l$ for $k=0,1,2, \ldots$,

then $\lim _{t \rightarrow \infty} D\left(U\left(t_{0}, U_{0}\right)(t), \bar{U}(t)\right)=0$.
Proof. Let $t \in I_{k}$ for some nature number $k$. Note that $e_{A}\left(t, t_{k}^{+}\right)=e_{A}\left(t, \sigma\left(t_{k}\right)\right)$, and combining the exponential dichotomy and the hypothesis (ii), one has

$$
\begin{aligned}
D(U( & \left.\left.t_{0}, U_{0}\right)(t), \bar{U}(t)\right) \\
= & D\left(V_{k}(t), \bar{U}(t)\right)=\left\|V_{k}(t)-{ }_{g} \bar{U}(t)\right\| \\
\leq & \left|e_{A}\left(t, t_{k}^{+}\right)\right|\left\|\Phi_{k}\right\| \\
& +\int_{t_{k}}^{t}\left|e_{A}(t, \sigma(\tau))\right|\left\|\left[F\left(\tau, V_{k}\right)-{ }_{g} F(\tau, \bar{U})\right]\right\| \Delta \tau \\
& +\sum_{t_{j}<t}\left|e_{A}^{0}(t) e_{\ominus A^{*}}^{0}\left(t_{j}^{+}\right)\right|\left\|H_{j}\right\| \\
\leq & k e_{\ominus a}\left(t, \sigma\left(t_{0}\right)\right)\left\|\Phi_{k}\right\| \\
& +\int_{t_{k}}^{t} k e_{\ominus a}(t, \sigma(\tau)) h(\tau) D\left(V_{k}(\tau), \bar{U}(\tau)\right) \Delta \tau \\
& +k \sum_{t_{j}<t} e_{\ominus a}\left(t, \sigma\left(t_{j}\right)\right)\left\|H_{j}\right\|
\end{aligned}
$$

and therefore, in view of (H2),

$$
\begin{aligned}
e_{a}(t, 0) & D\left(U\left(t_{0}, U_{0}\right)(t), \bar{U}(t)\right) \\
\leq & k e_{a}\left(\sigma\left(t_{0}\right), 0\right)\left\|\Phi_{k}\right\|+k\|\mathscr{H}\|_{\infty} \sum_{t_{j}<t} e_{a}\left(\sigma\left(t_{j}\right), 0\right) \\
& +\int_{t_{k}}^{t} k e_{a}(\sigma(\tau), 0) h(\tau) D\left(U\left(t_{0}, U_{0}\right)(\tau), \bar{U}(\tau)\right) \Delta \tau \\
\leq & k e_{a}\left(\sigma\left(t_{0}\right), 0\right)\left\|\Phi_{k}\right\|+\frac{k}{1-\chi}\|\mathscr{H}\|_{\infty} \\
& +\int_{t_{k}}^{t} k(1+a \mu(\tau)) h(\tau) e_{a}(\tau, 0) \\
& \times D\left(U\left(t_{0}, U_{0}\right)(\tau), \bar{U}(\tau)\right) \Delta \tau .
\end{aligned}
$$

In view of Gronwall's inequality ([40, Theorem 6.4]), we obtain

$$
\begin{aligned}
e_{a}(t, 0) & D\left(U\left(t_{0}, U_{0}\right)(t), \bar{U}(t)\right) \\
& \leq l_{k}+l_{k} \int_{t_{k}}^{t} e_{p}(t, \sigma(\tau)) p(\tau) \Delta \tau \\
& \leq l_{k}+l_{k} \int_{t_{k}}^{t} p(\tau) e_{p}(t, 0) \Delta \tau,
\end{aligned}
$$

where $p(\tau)=k(1+a \mu(\tau)) h(\tau), l_{k}=k e_{a}\left(\sigma\left(t_{0}\right), 0\right)\left\|\Phi_{k}\right\|+(k /(1-$ $\chi))\|\mathscr{H}\|_{\infty}$. From this, it follows that

$$
\begin{aligned}
& D\left(U\left(t_{0}, U_{0}\right)(t), \bar{U}(t)\right) \\
& \quad \leq l_{k} e_{\ominus a}(t, 0)+l_{k} e_{p \ominus a}(t, 0) \int_{t_{k}}^{t} p(\tau) \Delta \tau
\end{aligned}
$$


which guarantees that $D\left(U\left(t_{0}, U_{0}\right)(t), \bar{U}(t)\right) \rightarrow 0$ as $t \rightarrow \infty$ and the proof is complete.

We are now in a position to formulate the stability criteria for the null solution of ISDE (51). Let us first define the stability of trivial solution.

Definition 22. Let $U(t)=U\left(t_{0}, U_{0}\right)(t)$ be any solution of ISDE (51). Then the trivial solution $U(t) \equiv\{0\}$ is said to be stable if, for each $\epsilon>0$ and $t_{0} \in \mathbb{T}_{+}$, there exists a $\delta=\delta\left(t_{0}, \epsilon\right)>0$ such that $D\left(U_{0},\{0\}\right)<\delta$ implies that $D(U(t),\{0\})<\epsilon, t \in \mathbb{T}_{+}$.

Theorem 23. Under the assumption of Theorem 21, the trivial solution of ISDE (51) is stable.

Proof. From the above arguments we see that any solution of ISDE (51) can be indicated by



We first consider the case of $t \in\left[0, t_{0}\right]_{\mathbb{T}_{+}}$. As an analogy of the proof of Theorem 21, we have obtained

$$
e_{a}(t, 0) D(U(t),\{0\}) \leq l_{0}+l_{0} e_{p}(t, 0) \int_{0}^{t_{0}} p(\tau) \Delta \tau,
$$

where $p(\tau)=k(1+a \mu(\tau)) h(\tau), l_{0}=k e_{a}\left(\sigma\left(t_{0}\right), 0\right) D\left(U_{0},\{0\}\right)$. For any $\epsilon>0$, choose

$$
\delta_{1}=\epsilon\left[k e_{a}\left(\sigma\left(t_{0}\right), 0\right)\left(1+e_{a}\left(t_{0}, 0\right) \int_{0}^{t_{0}} p(\tau) \Delta \tau\right)\right]^{-1},
$$

and we have $D(U(t),\{0\})<\epsilon$ whenever $D\left(U_{0},\{0\}\right)<\delta_{1}$.

If $t \in\left(t_{0}, \infty\right)_{\mathbb{T}_{+}}$, by the exponential dichotomy we have

$$
\begin{aligned}
& D(U(t),\{0\}) \\
& \leq D(W(t),\{0\})+e_{a}(0, t) D\left(U_{0},\{0\}\right) \\
& \leq \int_{t_{0}}^{t}\left|e_{A}^{0}(t) e_{\ominus A^{*}}^{0}(\sigma(\tau))\right| D(F(\tau, U), F(t,\{0\})) \Delta \tau \\
&+e_{a}(0, t) D\left(U_{0},\{0\}\right) \\
& \leq \int_{t_{0}}^{t} k e_{\ominus a}(t, \sigma(\tau)) h(\tau) D(U(\tau),\{0\}) \Delta \tau \\
&+e_{a}(0, t) D\left(U_{0},\{0\}\right)
\end{aligned}
$$

and therefore

$$
\begin{aligned}
e_{a}(t, 0) & D(U(t),\{0\}) \\
\leq & \int_{t_{0}}^{t} k e_{a}(\sigma(\tau), 0) h(\tau) D(U(\tau),\{0\}) \Delta \tau+D\left(U_{0},\{0\}\right) \\
& =\int_{t_{0}}^{t} p(\tau) e_{a}(\tau, 0) D(U(\tau),\{0\}) \Delta \tau+D\left(U_{0},\{0\}\right) .
\end{aligned}
$$

Gronwall's inequality again implies that

$$
\begin{aligned}
& e_{a}(t, 0) D(U(t),\{0\}) \\
& \quad \leq D\left(U_{0},\{0\}\right) \int_{t_{0}}^{t} p(\tau) e_{p}(t, \sigma(\tau)) \Delta \tau+D\left(U_{0},\{0\}\right),
\end{aligned}
$$

which implies that

$$
D(U(t),\{0\}) \leq D\left(U_{0},\{0\}\right)\left(1+\int_{t_{0}}^{\infty} p(\tau) \Delta \tau\right) .
$$

Taking $\delta_{2}=\epsilon\left(1+\int_{t_{0}}^{\infty} p(\tau) \Delta \tau\right)^{-1}$, we have $D(U(t),\{0\})<\epsilon$ if $D\left(U_{0},\{0\}\right)<\delta_{2}$. Let $\delta=\min \left\{\delta_{1}, \delta_{2}\right\}$. Then $D\left(U_{0},\{0\}\right)<\delta$ implies that $D(U(t),\{0\})<\epsilon, t \in \mathbb{T}_{+}$. This proof is complete.

\section{Examples}

In this section we present several examples to illustrate the applicability of the results involved in the above sections.

Example 1. Consider the set dynamic equation

$$
\begin{gathered}
\Delta_{g} U(t)=A U(t)+F(t), \quad t \in \mathbb{T}_{+}, t \neq t_{k}, \\
U\left(t_{k}^{+}\right)=B U\left(t_{k}\right), \quad k=0,1,2 \ldots, \\
U\left(t_{0}\right)=U_{0},
\end{gathered}
$$

where $A=\left(\begin{array}{cc}-\lambda & 0 \\ 0 & -\lambda\end{array}\right), \lambda>0$, and $-\lambda \in \mathscr{R}_{1}^{+}, F(t)=f(t) C$ with $f(t)=\left(\begin{array}{c}\sin \sqrt{3} t \\ \cos \sqrt{2} t\end{array}\right), C \subset \mathbb{R}$ a bounded subset, $B$ is a $2 \times$ 2-constant-valued matrix, and $t_{k}=(k+1) t_{0} \in \mathbb{T}_{+}$for $k=$ $0,1,2, \ldots$

Conclusion. Equation (106) has a unique bounded solution

$$
U(t)=\left\{\begin{array}{rr}
e_{-\lambda}\left(t, t_{0}\right) I_{2} U_{0} & \\
+\int_{t_{0}}^{t} e_{-\lambda}(t, \sigma(\tau)) I_{2} f(\tau) C \Delta \tau, & t \in\left[0, t_{0}\right]_{\mathbb{T}_{+}}, \\
\int_{t_{0}}^{t} e_{-\lambda}(t, \sigma(\tau)) I_{2} f(\tau) C \Delta \tau, & t \in\left(t_{0}, \infty\right)_{\mathbb{T}_{+}} \\
\text {with } I_{2}=\left(\begin{array}{ll}
1 & 0 \\
0 & 1
\end{array}\right) .
\end{array}\right.
$$


Proof. From [40, Theorem 5.35] it is easy to see that the homogeneous equation of (106) admits an exponential dichotomy with the constants $k=a=1$, the projection $P=I_{2}$, and the fundamental matrix $e_{A}^{0}(t)=e_{-\lambda}(t, 0) I_{2}$. Thus, the condition $(\mathrm{H} 1)$ is naturally satisfied. Let $B C_{0}=$ $\left\{U \in B C\left(\mathbb{T}_{+}, K_{c}^{2}\right): U_{t_{k}^{+}}=B\left(U\left(t_{k}\right)\right)\right\}$. Now Remark 17 guarantees that the existence of the unique bounded solution $U$ to (106) and the expression of $U$ are immediately obtained by Corollary 16.

Example 2. Let us consider an interval-valued dynamic equation

$$
\begin{gathered}
\Delta_{g} U(t)=-p(t) U(t)+F(t, U), \\
t \neq t_{k} \quad(k=0,1,2, \ldots), \\
U_{t_{k}^{+}}=J_{k} U\left(t_{k}\right)+H_{k}, \quad k=0,1,2, \ldots, \\
U\left(t_{0}\right)=U_{0},
\end{gathered}
$$

where $U(t)=\left[u^{-}(t), u^{+}(t)\right] \in \mathbb{}, F(t, U)=$



Conclusion. If (ii) of Theorem 21 holds, $e_{1}\left(\sigma\left(t_{k}\right), \sigma\left(t_{k+1}\right)\right) \leq$ $\chi<1$ for $k=0,1,2, \ldots$, and the following hypotheses hold:

(I) $p \in C_{r d}\left(\mathbb{T}_{+}, \mathbb{R}_{+}\right), \inf _{t \in \mathbb{T}_{+}} p(t)>0$ and $-p \in \mathscr{R}_{1}^{+}$;

(II) $f^{-}, f^{+} \in P C\left(\mathbb{T}_{+} \times \mathbb{\square}, \mathbb{R}\right)$;

(III) $f_{1}, f_{2} \in \operatorname{Lip}(h)$,

then the following results are valid.

(i) Let $U_{k}(t)=\left[u_{k}^{-}(t), u_{k}^{+}(t)\right]$ be a solution of (90) with $\Phi_{k} \in \mathbb{}\left(t \in I_{k}, k=0,1,2, \ldots\right)$. For any $\varepsilon>0$, there exists the natural number $k$ such that

$$
\begin{aligned}
& \left|\min \left\{u_{k}^{-}(t)-\bar{u}^{-}(t), u_{k}^{+}(t)-\bar{u}^{+}(t)\right\}\right|<\varepsilon, \\
& \left|\max \left\{u_{k}^{-}(t)-\bar{u}^{-}(t), u_{k}^{+}(t)-\bar{u}^{+}(t)\right\}\right|<\varepsilon
\end{aligned}
$$

for $t \in I_{k}$, where $\bar{U}(t)=\left[\bar{u}^{-}(t), \bar{u}^{+}(t)\right]$ is a solution of (108).

(ii) The trivial solution of (108) is stable.

Proof. Clearly, the homogeneous equation $\Delta_{g} U(t)=$ $p(t) U(t)$ admits an exponential dichotomy with constants $k=a=1$, the projection $P=I$, and the fundamental matrix $e_{-p}^{0}(t)$; that is, the hypothesis $(\mathrm{H} 1)$ of Theorem 21 is valid. Our assumption guarantees that $(\mathrm{H} 2)$ is also valid.

For $\left(t, U_{1}\right),\left(t, U_{2}\right) \in \mathbb{T}_{+} \times \mathbb{\square}$, set $f_{1}^{-}=f^{-}\left(t, U_{1}\right), f_{2}^{-}=$ $f^{-}\left(t, U_{2}\right)$ and we similarly stipulate $f^{+}$. Since $f_{1}, f_{2} \in \operatorname{Lip}(h)$, by means of [33] we have

$$
\begin{aligned}
& D\left(F\left(t, U_{1}\right), F\left(t, U_{2}\right)\right) \\
& =\max \left\{\left|\min \left\{f_{1}^{-}-f_{2}^{-}, f_{1}^{+}-f_{2}^{+}\right\}\right|,\right. \\
& \left.\quad\left|\max \left\{f_{1}^{-}-f_{2}^{-}, f_{1}^{+}-f_{2}^{+}\right\}\right|\right\} \\
& \leq D\left(U_{1}, U_{2}\right) h(t) .
\end{aligned}
$$

This implies that $F \in \operatorname{Lip}(h)$. In virtue of Theorem 21 , for any $\varepsilon>0$, there exists a $T>0$ such that

$$
D\left(U\left(t_{0}, U_{0}\right)(t), \bar{U}(t)\right)<\varepsilon
$$

for any $t>T$, where $U\left(t_{0}, U_{0}\right)$ is given as in (92). By means of (92), there exists some natural number $k$ such that $t \in I_{k}$ when $t>T$ is given. Thus, $U\left(t_{0}, U_{0}\right)(t)=U_{k}(t)$. Hence,

$$
\begin{aligned}
D(U & \left.\left(t_{0}, U_{0}\right)(t), \bar{U}(t)\right) \\
& =D\left(U_{k}(t), \bar{U}(t)\right)=\max \left\{\left|\mathcal{u}^{-}(t)\right|,\left|u^{+}(t)\right|\right\}<\varepsilon,
\end{aligned}
$$

where $u^{-}(t)=\min \left\{u_{k}^{-}(t)-\bar{u}^{-}(t), u_{k}^{+}(t)-\bar{u}^{+}(t)\right\}, u^{+}=$ $\max \left\{u_{k}^{-}(t)-\bar{u}^{-}(t), u_{k}^{+}(t)-\bar{u}^{+}(t)\right\}$. (i) is valid.

(ii) is an immediate result of Theorem 23 .

In order to apply our results to fuzzy problems to obtain the existence and stability of bounded solutions to fuzzy dynamic equations on a time scale, we need some terse memories for fuzzy theory for its analogy in $\mathbb{R}$ referring to [43]. A function $u: \mathbb{T} \rightarrow[0,1]$ is called a fuzzy number on time scale if it satisfies the following properties:

(i) $u$ is normal; that is, there exists $s_{0} \in \mathbb{T}$ such that $u\left(s_{0}\right)=1$,

(ii) $u$ is a convex fuzzy set on $\mathbb{T}$ (i.e., $u(t a+(1-t) b) \geq$ $\min \{u(a), u(b)\}, \forall t \in[0,1], a, b \in \mathbb{T}, t a+(1-t) b \in \mathbb{T})$,

(iii) $u$ is upper semicontinuous on $\mathbb{T}$,

(iv) $[u]^{0}=\overline{\{s \in \mathbb{T}: u(s)>0\}}$ is compact.

Let $\mathbb{T}_{F}$ denote the space of fuzzy numbers. For $0<r \leq 1$, the set $[u]^{r}=\{s \in \mathbb{T}: u(s) \geq r\}$ is called the $r$-level set of $u$. Obviously $[u]^{r}$ is a compact interval of $\mathbb{T}$ if $u \in \mathbb{T}_{F}$ for all $r \in[0,1]$. The notation $[u]^{r}=\left[u_{r}^{-}, u_{r}^{+}\right]_{\mathbb{T}}$ denotes explicitly the $r$-level set of $u$. we refer to $u^{-}$and $u^{+}$as the lower and upper branches of $u$, respectively. For any $u, v \in \mathbb{T}_{F}$, the metric structure is given by the Hausdorff distance

$$
D_{f}(u, v)=\sup _{r \in[0,1]} \max \left\{\left|u_{r}^{-}-v_{r}^{-}\right|,\left|u_{r}^{+}-v_{r}^{+}\right|\right\} .
$$

Thus $\left(\mathbb{T}_{F}, D_{f}\right)$ is a complete metric space.

Consider the existence and stability of bounded solutions for the impulsive fuzzy differential equation on $\mathbb{T}$

$$
\begin{gathered}
\Delta_{g} Y(t)=-p(t) Y(t)+\tilde{2}_{\ominus 1}(\sigma(t), 0) Y(t)^{2}, \\
t \neq t_{k}(k=0,1,2, \ldots), \\
Y_{t_{k}^{+}}=J_{k} Y\left(t_{k}\right)+H_{k}, \quad k=0,1,2, \ldots, \\
Y(0)=Y_{0},
\end{gathered}
$$

where $p, t_{k}, J_{k}, H_{k}$ satisfy the hypotheses of Example 2, respectively. The $r$-level set of fuzzy number $\tilde{2}$ is [2] ${ }^{r}=[r+$ $1,3-r]$ for all $r \in[0,1]$. Let $F(t, Y)=\tilde{2} e_{\ominus 1}(\sigma(t), 0) Y(t)^{2}$. Then $r$-level set of $F(t, Y)$ is $[F(t, Y)]^{r}=e_{\ominus 1}(\sigma(t), 0)[(r+$ $\left.1)\left(Y_{r}^{-}\right)^{2},(3-r)\left(Y_{r}^{+}\right)^{2}\right]$. Further, we have the following. 
Theorem 24. Let $\mathbb{T}_{F}^{1 / 6}=\left\{X \in P C^{1}: D_{0}(X,\{0\}) \leq 1 / 6\right\}$. If $p, t_{k}, J_{k}, H_{k}$ satisfy the hypotheses of Example 2, then fuzzy dynamic equation (114) has a bounded solution which satisfies the results of Example 2.

Proof. Note that $\mathbb{T}_{F}^{1 / 6}$ is a metric space with respect to $D_{f}$. In virtue of Theorems 21 and 23, it suffices to prove $F(t, Y)=$ $\widetilde{2} e_{\ominus 1}(\sigma(t), 0) Y(t)^{2} \in \operatorname{Lip}(h)$. In fact, for any $X, Y \in \mathbb{T}_{F}^{1 / 6}$, we have

$$
\begin{aligned}
D_{f}\left([F(t, X)]^{r},[F(t, Y)]^{r}\right) & \\
= & D_{f}\left(e_{\ominus 1}(\sigma(t), 0)\left[(r+1)\left(X_{r}^{-}\right)^{2},(3-r)\left(X_{r}^{+}\right)^{2}\right]\right. \\
& \left.e_{\ominus 1}(\sigma(t), 0)\left[(r+1)\left(Y_{r}^{-}\right)^{2},(3-r)\left(Y_{r}^{+}\right)^{2}\right]\right) \\
= & e_{\ominus 1}(\sigma(t), 0) \max \left\{(1+r)\left|\left(X_{r}^{-}\right)^{2}-\left(Y_{r}^{-}\right)^{2}\right|\right. \\
\leq & 3 e_{\ominus 1}(\sigma(t), 0)\left|X_{r}^{+}+Y_{r}^{+}\right| \\
& \times \max \left\{\left|X_{r}^{-}-Y_{r}^{-}\right|,\left|X_{r}^{+}-Y_{r}^{+}\right|\right\} \\
\leq & 3 e_{\ominus 1}(\sigma(t), 0)\left|X_{r}^{+}+Y_{r}^{+}\right| D_{f}(X, Y) \\
\leq & e_{\ominus 1}(\sigma(t), 0) D_{f}(X, Y) .
\end{aligned}
$$

Let $h(t)=e_{\ominus 1}(\sigma(t), 0)$. Then $h: \mathbb{T}_{+} \rightarrow \mathbb{R}_{+}$satisfies

$$
(1+\mu(t)) h(t)=\frac{1}{1+\mu(t) \cdot \ominus 1} e_{\ominus 1}(\sigma(t), 0)=e_{\ominus 1}(t, 0) .
$$

This implies that $(1+\mu(t)) h(t) \leq 1$ and $\int_{0}^{\infty}(1+\mu(\tau)) h(\tau) \Delta \tau<$ $\infty$. Consequently, $F \in \operatorname{Lip}(h)$. This proof is complete.

\section{Conflict of Interests}

The authors declare that there is no conflict of interests regarding the publication of this paper.

\section{Acknowledgment}

This research is supported by Natural Science Foundation of Zhejiang Province (LY12A01002).

\section{References}

[1] R. P. Agarwal and D. O'Regan, "Existence for set differential equations via multivalued operator equations," Differential Equations and Applications, vol. 5, pp. 1-5, 2007.

[2] B. Ahmad and S. Sivasundaram, "Dynamics and stability of impulsive hybrid setvalued integro-differential equations with delay," Nonlinear Analysis, Theory, Methods and Applications, vol. 65, no. 11, pp. 2082-2093, 2006.

[3] B. Ahmad and S. Sivasundaram, "Stability in terms of two measures for setvalued perturbed impulsive delay differential equations," Communications in Applied Analysis, vol. 12, no. 1, pp. 57-68, 2008.

[4] B. Ahmad and S. Sivasundaram, "OøoStability of impulsive hybrid setvalued differential equations with delay by perturbing lyapunov functions," Communications in Applied Analysis, vol. 12, no. 2, pp. 137-146, 2008.

[5] N. V. Hoa and N. D. Phu, "On maximal and minimal solutions for set-valued differential equations with feedback control," Abstract and Applied Analysis, vol. 2012, Article ID 816218, 11 pages, 2012.

[6] A. V. Plotnikov and A. V. Arsirii, "Piecewise constant control set systems," American Journal of Computational and Applied Mathematics, vol. 1, no. 2, pp. 89-93, 2011.

[7] A. V. Plotnikov and N. V. Skripnik, "Set-valued differential equations with generalized derivative," Journal of Advanced Research in Pure Mathematics, vol. 3, no. 1, pp. 144-160, 2011.

[8] A. V. Plotnikov and N. V. Skripnik, "Existence and uniqueness theorem for fuzzy integral equation," International Journal of Mathematical Sciences and Applications, vol. 1, no. 1, pp. 1-5, 2013.

[9] M. T. Malinowski, "On set differential equations in Banach spaces-a second type Hukuhara differentiability approach," Applied Mathematics and Computation, vol. 219, no. 1, pp. 289305, 2012.

[10] M. T. Malinowski, "Second type Hukuhara differentiable solutions to the delay set-valued differential equations," Applied Mathematics and Computation, vol. 218, no. 18, pp. 9427-9437, 2012.

[11] M. T. Malinowski, "Interval Cauchy problem with a second type Hukuhara derivative," Information Sciences, vol. 213, no. 23, pp. 94-105, 2012.

[12] M. T. Malinowski, "ItÔ type stochastic fuzzy differential equations with delay," Systems and Control Letters, vol. 61, no. 6, pp. 692-701, 2012.

[13] M. T. Malinowski, "Some properties of strong solutions to stochastic fuzzy differential equations," Information Sciences, vol. 248, no. 23, pp. 50-67, 2013.

[14] M. T. Malinowski, "On a new set-valued stochastic integral with respect to semimartingales and its applications," Journal of Mathematical Analysis and Applications, vol. 408, no. 2, pp. 669-680, 2013.

[15] M. T. Malinowski, "Approximation schemes for fuzzy stochastic integral equations," Applied Mathematics and Computation, vol. 219, no. 24, pp. 11278-11290, 2013.

[16] J. Jiang, C. Li, and H. Chen, "Existence of solutions for set differential equations involving causal operator with memory in Banach space," Journal of Applied Mathematics and Computing, vol. 41, no. 1-2, pp. 183-196, 2013.

[17] V. Laksmikantham, "Set differential equations versus fuzzy differential equations," Applied Mathematics and Computation, vol. 164, no. 2, pp. 277-297, 2005.

[18] V. Lakshmikatham, T. G. Bhaskar, and J. V. Devi, Theory of Set Differential Equations in Metric Spaces, Cambridge Scientic, Cambridge, UK, 2006.

[19] L. Li and S. Hong, "Exponential stability for set dynamic equations on time scales," Journal of Computational and Applied Mathematics, vol. 235, no. 17, pp. 4916-4924, 2011.

[20] F. A. Mcrae and J. V. Devi, "Impulsive set differential equations with delay," Applicable Analysis, vol. 84, no. 4, pp. 329-341, 2005.

[21] S. H. Hong, "Differentiability of multivalued functions on time scales and applications to multivalued dynamic equations," 
Nonlinear Analysis, Theory, Methods and Applications, vol. 71, no. 9, pp. 3622-3637, 2009.

[22] S. H. Hong, "Stability criteria for set dynamic equations on time scales," Computers and Mathematics with Applications, vol. 59, no. 11, pp. 3444-3457, 2010.

[23] S. H. Hong and J. Liu, "Phase spaces and periodic solutions of set functional dynamic equations with infinite delay," Nonlinear Analysis, Theory, Methods and Applications, vol. 74, no. 9, pp. 2966-2984, 2011.

[24] J. Vasundhara Devi and Ch. Appala Naidu, "Stability results for set differential equations Involving causal operators with memory," European Journal of Pure and Applied Mathematics, vol. 5, no. 2, pp. 187-196, 2012.

[25] M. Malinowski, M. Michta, and J. Sobolewska, "Set-valued and fuzzy stochastic differential equations driven by semimartingales," Nonlinear Analysis: Theory, Methods \& Applications, vol. 79, no. 5, pp. 204-220, 2013.

[26] N. Tu and T. Tung, "Stability of set differential equations and applications," Nonlinear Analysis, Theory, Methods and Applications, vol. 71, no. 5-6, pp. 1526-1533, 2009.

[27] V. Ghaffari and P. Karimaghaee, "Adaptive observer-based stabilizer for a class of nonlinear systems and its application to chaos control," International Journal of Control Science and Engineering, vol. 2, no. 2, pp. 1-6, 2012.

[28] V. Lupulescuc, "Hukuhara differentiability of interval-valued functions and interval differential equations on time scales," Information Sciences, vol. 248, no. 21, pp. 50-67, 2013.

[29] M. Hukuhara, "Sur l'aplication semicontinuone dont la valeur est un compact convexe," Funkcialaj Ekvacioj, vol. 10, pp. 43-68, 1967.

[30] S. Seikkala, "On the fuzzy initial value problem," Fuzzy Sets and Systems, vol. 24, no. 3, pp. 319-330, 1987.

[31] B. Bede and S. G. Gal, "Almost periodic fuzzy-number-valued functions," Fuzzy Sets and Systems, vol. 147, no. 3, pp. 385-403, 2004.

[32] B. Bede and S. G. Gal, "Generalizations of the differentiability of fuzzy-number-valued functions with applications to fuzzy differential equations," Fuzzy Sets and Systems, vol. 151, no. 3, pp. 581-599, 2005.

[33] L. Stefanini and B. Bede, "Generalized Hukuhara differentiability of interval-valued functions and interval differential equations," Nonlinear Analysis, Theory, Methods and Applications, vol. 71, no. 3-4, pp. 1311-1328, 2009.

[34] Y. Chalco-Cano, H. Román-Flores, and M. D. Jiménez-Gamero, "Generalized derivative and $\pi$-derivative for set-valued functions," Information Sciences, vol. 181, no. 11, pp. 2177-2188, 2011.

[35] D. D. Bainov, S. I. Kostadinov, and P. P. Zabreiko, "Exponential dichotomy of linear impulsive differential equations in a Banach space," International Journal of Theoretical Physics, vol. 28, no. 7, pp. 797-814, 1989.

[36] D. Zhang and B. Dai, "Existence of solutions for nonlinear impulsive differential equations with Dirichlet boundary conditions," Mathematical and Computer Modelling, vol. 53, no. 5-6, pp. 1154-1161, 2011.

[37] J. Yang and B. Xie, "New results on impulsive functional differential equations with infinite delays," Abstract and Applied Analysis, vol. 2013, Article ID 132619, 8 pages, 2013.

[38] G. L. Zhang, M. H. Song, and M. Z. Liu, "Exponential stability of impulsive delay differential equations," Abstract and Applied Analysis, vol. 2013, Article ID 938027, 6 pages, 2013.
[39] S. Hilger, "Analysis on measure chains- a unified approach to continuous and discrete calculus," Results in Mathematics, vol. 18, no. 1-2, pp. 18-56, 1990.

[40] M. Bohner and A. Peterson, Dynamic Equations on Time Scales, an Introduction with Applications, Birkhäuser, Boston, Mass, USA, 2001.

[41] L. Stefanini, "A generalization of Hukuhara difference for interval and fuzzy arithmetic," in Soft Methods for Handling Variability and Imprecision, D. Dubois, M. A. Lubiano, H. Prade, M. A. Gil, P. Grzegorzewski, and O. Hryniewicz, Eds., vol. 48 of Series on Advances in Soft Computing, Springer, New York, NY, USA, 2008, http://econpapers.repec.org/RAS/pst233.htm .

[42] S. H. Hong and Y. Peng, "Almost periodicity of set-valued functions and set dynamic equations on time scales," Information Sciences. In press.

[43] A. Khastan, J. J. Nieto, and R. Rodríguez-López, "Variation of constant formula for first order fuzzy differential equations," Fuzzy Sets and Systems, vol. 177, no. 1, pp. 20-33, 2011. 


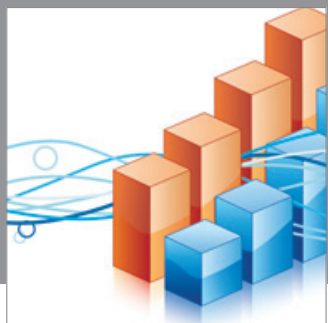

Advances in

Operations Research

mansans

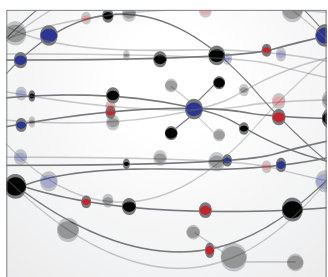

The Scientific World Journal
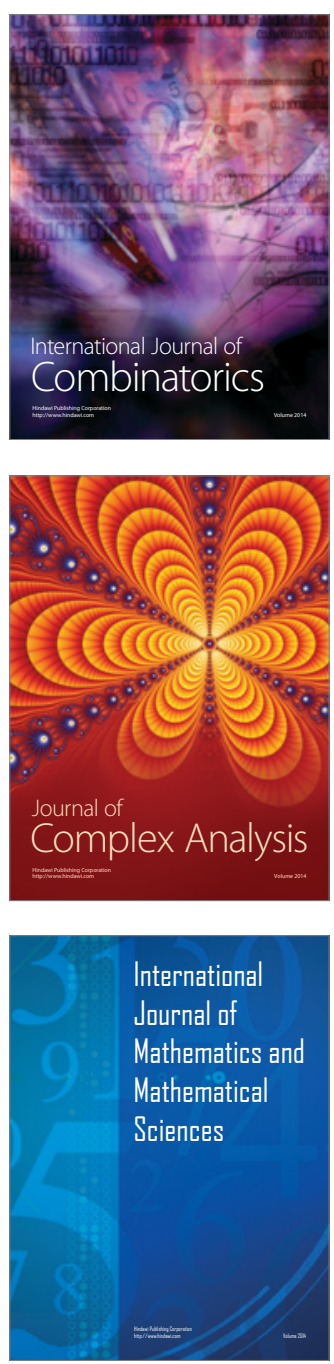


Submit your manuscripts at http://www.hindawi.com
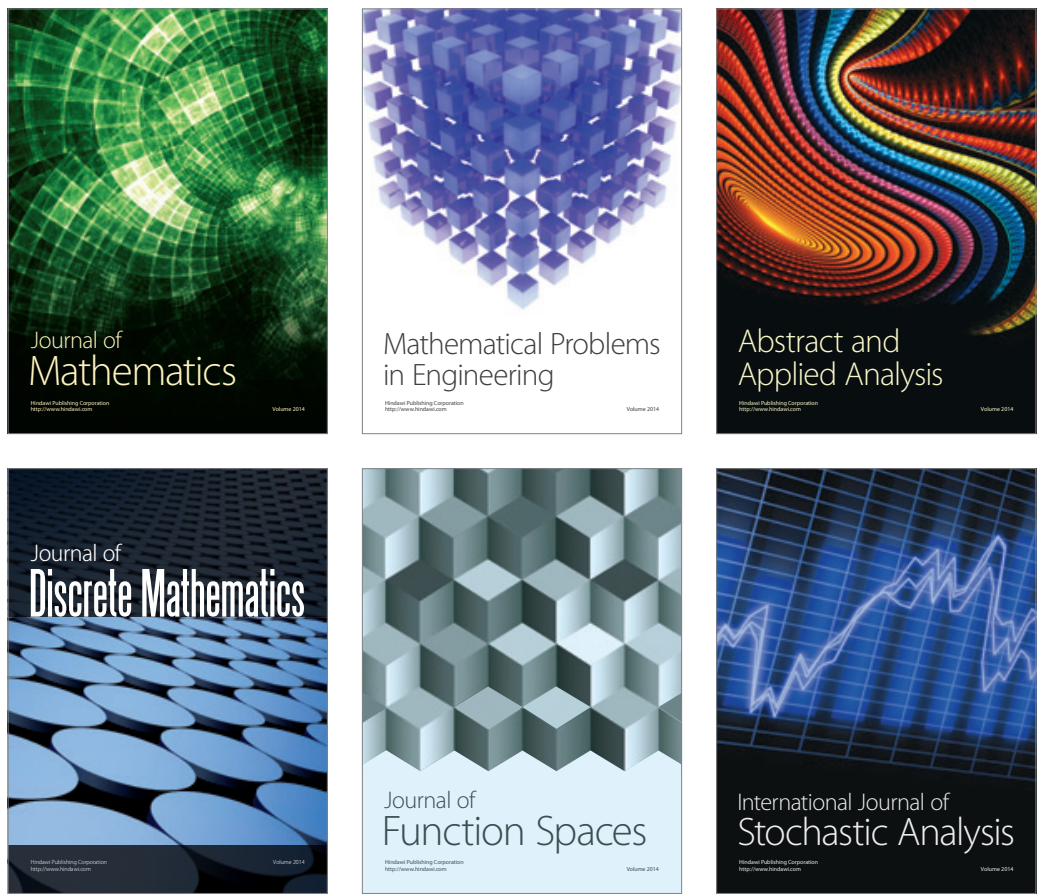

Journal of

Function Spaces

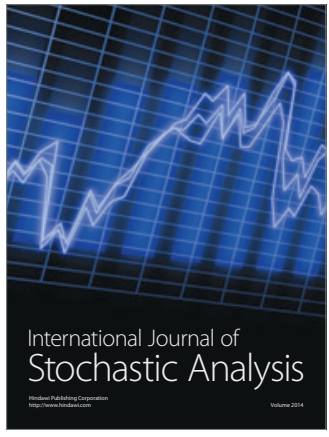

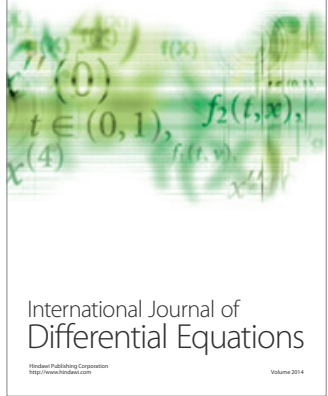
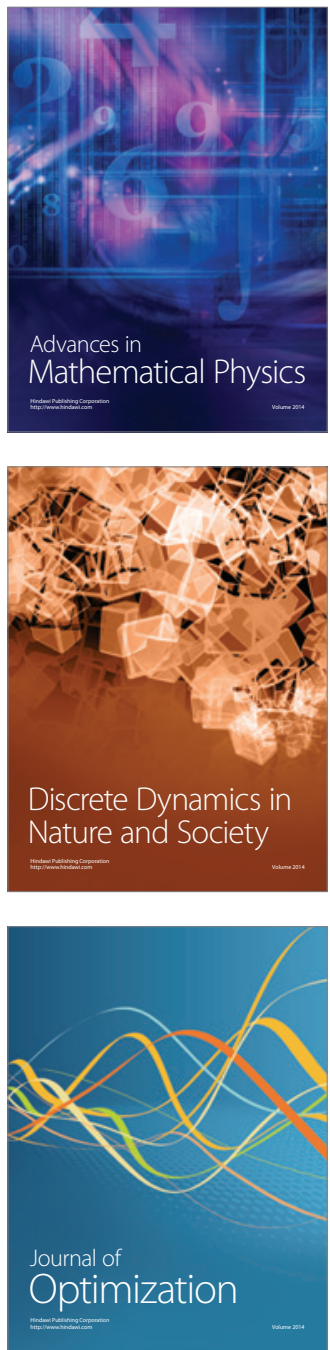